\title{
Quantum Nuclear Motion of Helium and Molecular Nitrogen Clusters in Carbon Nanotubes
}

\author{
Andreas W. Hauser, ${ }^{\dagger}$ (৫) Alexander O. Mitrushchenkov, ${ }^{\ddagger}$ and María Pilar de Lara-Castells*,§® \\ ${ }^{\dagger}$ Graz University of Technology, Institute of Experimental Physics, Petersgasse 16, 8010 Graz, Austria \\ ${ }^{\ddagger}$ Université Paris-Est, Laboratoire Modélisation et Simulation Multi Echelle, MSME UMR 8208 CNRS, 5 bd Descartes, 77454 \\ Marne-la-Vallée, France \\ ${ }^{\S}$ Instituto de Física Fundamental (C.S.I.C.), Serrano 123, E-28006 Madrid, Spain
}

Supporting Information

ABSTRACT: We study the quantum nuclear motion of $N^{4} \mathrm{He}$ atoms or $\mathrm{N} \mathrm{N}_{2}$ molecules $(N<4)$ confined in carbon nanotubes using an ad hoc-developed nuclear wave function-based approach. Density functional theory (DFT)-based symmetryadapted perturbation theory is used to derive parameters for a new pairwise potential model describing the gas adsorption to carbon materials. The predicted nuclear motion of $\mathrm{He}$ atoms inside a confining potential is directly compared to probability densities obtained by orbital-free He-DFT theory. The interaction of small clusters of adsorbates is also studied via a combination of both the discrete atomic and the continuous density approaches. Our results agree well with previously reported experimental and theoretical studies and provide new physical insights into the very different quantum confinement effects depending on the diameter of the carbon nanotubes and the impact of quantum phenomena on the gas storage capabilities at low temperatures.

\section{INTRODUCTION}

Carbon-based nanoporous materials and, in particular, carbon nanotubes (CNTs), occupy nowadays a pivotal role in nanoscience $^{1}$ and nanotechnology. ${ }^{2}$ High-surface areas and precisely tuned pores of these cylindrically deformed graphenelike materials make them useful for applications such as gas adsorption, in particular for the efficient storage of $\mathrm{H}_{2}$, improving conventional liquids or compressed gas technologies, the selective separation of light isotopes, ${ }^{3}$ and, more recently, as nanoreactors to synthesize and confine metal nanoparticles in quasi one-dimensional configurations. ${ }^{4}$

Understanding the role of nuclear quantum effects and intramolecular interactions in the adsorption of molecules in carbon nanotubes is deeply fundamental, being also a prerequisite in controlling the potential capacities of the nanotubes in, for instance, gas storage and isotope separation. Adsorption and diffusion processes through porous materials are most often studied by molecular dynamics simulations. For the case of light species, quantum zero point and tunneling effects can be significant. An interesting example is the ${ }^{3} \mathrm{He} /{ }^{4} \mathrm{He}$ separation via its diffusion through the nanopores of graphene membranes ${ }^{5,6}$ and graphdiyne. ${ }^{7,8}$

Very recently, Ohba has measured the adsorption of helium in carbon nanopores at very low temperatures of $2-5 \mathrm{~K}$, comparing it with that of molecular nitrogen. ${ }^{9}$ In contrast with the classical expectation based on kinetic diameters, while helium was found to be barely adsorbed through carbon

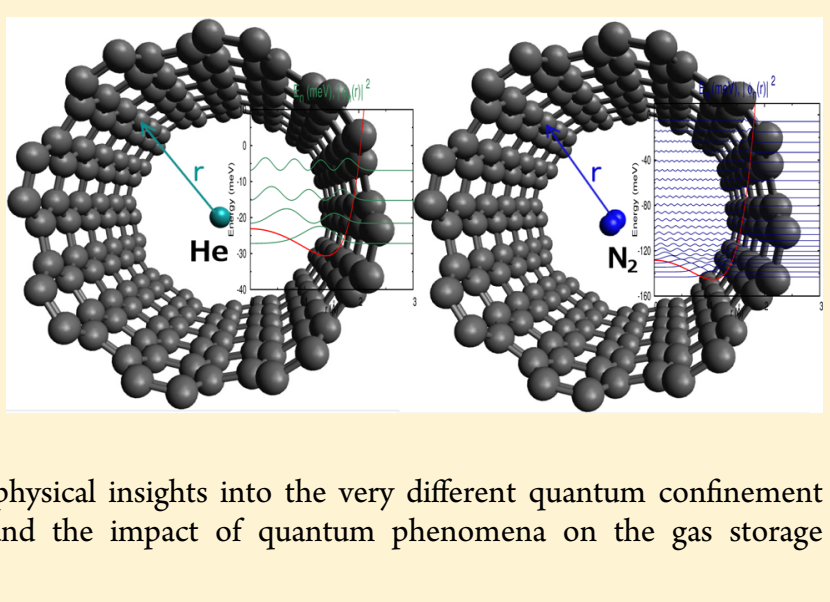

nanopores of diameter below $0.7 \mathrm{~nm}$, an unhampered diffusion was noticed for nitrogen molecules through smaller diameter nanopores. Using Path Integral Molecular Dynamics (PIMD) simulations with semiempirical potentials, ${ }^{9}$ the author attributed the experimental observations to the quantum behavior of helium in the low-temperature regime.

A key limitation in simulating adsorption processes is the selection of the adsorbate-CNT and adsorbate-adsorbate interaction potentials. Highly accurate potentials exist for adsorbate-adsorbate interactions and, in particular, for $\mathrm{He}-\mathrm{He}$ and $\mathrm{N}_{2}-\mathrm{N}_{2}$ pairs, ${ }^{10,11}$ based on very accurate ab initio calculations, accounting for post-BO relativistic and quantum electrodynamic effects, ${ }^{10}$ and are rigorously validated by experimental measurements. ${ }^{12,13}$ Although accurate adsorbate-CNT potentials are generally less available, modern intermolecular interaction theory along with efficient densityfitting techniques allow an ab initio characterization of van der Waals (vdW) interactions at the nanoscale. For example, recent options for accurate calculations of vdW adsorbate-surface interactions include the incremental method applied at the coupled-cluster level, ${ }^{14-18}$ embedded approaches, ${ }^{19}$ symmetryadapted perturbation theory, ${ }^{19-22}$ and mixed strategies

Received: December 24, 2016

Revised: January 28, 2017

Published: January 31, 2017 

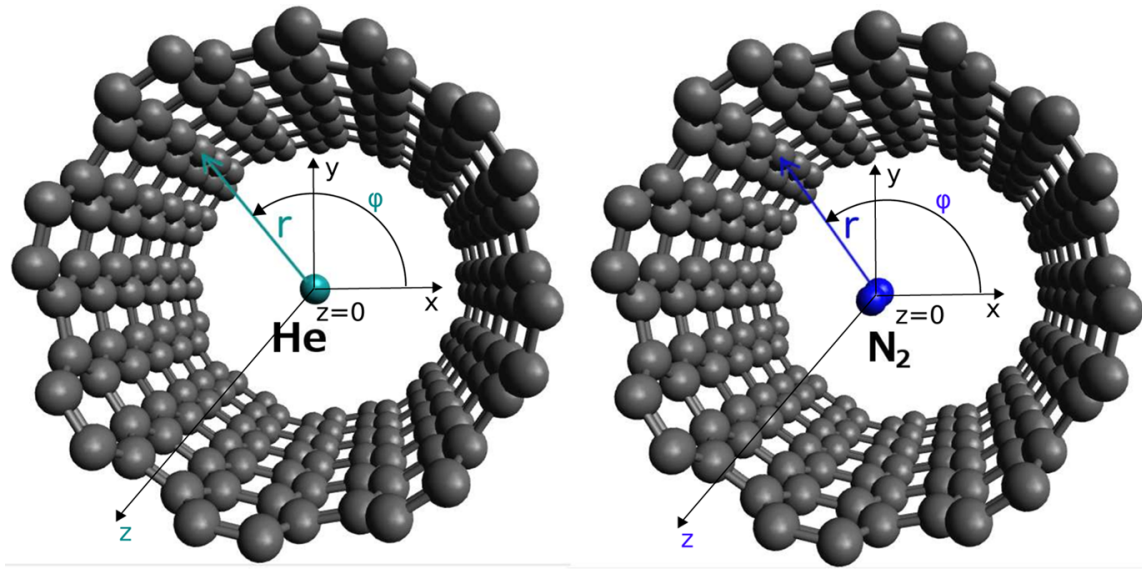

Figure 1. Figure illustrating the atomic structural models for ${ }^{4} \mathrm{He} / \mathrm{CNT}$ and $\mathrm{N}_{2} / \mathrm{CNT}$ systems. Gray spheres represent carbon atoms while sea-green and blue spheres stand for one ${ }^{4} \mathrm{He}$ atom and one $\mathrm{N}_{2}$ molecule inside a carbon nanotube with diameter of about $1.1 \mathrm{~nm}$. Cylindrical coordinates $(r$, $\phi, z)$ of the ${ }^{4} \mathrm{He}$ atom and the $\mathrm{N}_{2}$ mass center are also indicated. The $\mathrm{N}-\mathrm{N}$ bond is oriented along the $z$ axis, with the bond length fixed to the equilibrium value, $r_{\text {eq }}=1.098 \AA$.

combining nonperiodic ab initio and periodic DFT schemes. $^{15-18,23}$

In our recent theoretical study ${ }^{21}$ (referred to as Paper I in the following), the $\mathrm{He} / \mathrm{CNT}$ interaction potential was determined by extending the atom-bond potential model. ${ }^{24}$ To validate this model, ab initio calculations were carried out with DFTbased symmetry adapted perturbation theory [SAPT(DFT).$^{25,26}$ In this follow-up study, we present SAPT(DFT) interaction energies for both $\mathrm{He} / \mathrm{CNT}$ and $\mathrm{N}_{2} / \mathrm{CNT}$ systems using larger electronic basis sets than in Paper I. ${ }^{21}$ Moreover, a new pairwise potential model (PPM) is proposed, with model parameters extracted from the SAPT(DFT) dispersionless and dispersion interaction energies.

Another major difficulty in simulating adsorption in CNTs at the nanoscale is in accounting for the quantum nature of light adsorbates at very low temperatures. For the particular case of helium, ${ }^{21}$ this problem could be overcome by applying orbitalfree helium density functional theory at zero-temperature (the He-DFT method). ${ }^{27,28}$ Using this approach, a ${ }^{4} \mathrm{He}_{2000}$ droplet was described as a condensate with all the helium atoms in the same quantum state and the CNT was embedded within the droplet. This way, it was shown that the experimental result arises from the exceptionally high zero-point energy of helium and its tendency to form spatially separated layers upon adsorption at low temperatures. ${ }^{21}$ Furthermore, by averaging the helium density flooding into the immersed CNT, CNT diameter-dependent helium filling factors were determined which agree well with the experimental data. ${ }^{9}$

The helium layering structure in nanotubes reported in Paper $\mathrm{I}^{21}$ is also consistent with recent PIMC and quantum Monte Carlo (QMC) simulations of helium flow in carbon nanotubes ${ }^{29}$ and through $\mathrm{Si}_{3} \mathrm{~N}_{4}$ nanopores. ${ }^{30}$ An interesting difference between He-DFT and PIMC/QMC simulations is that the former indicates the formation of cavities of zero density within the tubes. ${ }^{21}$ However, zero radial densities have been reported in very recent QMC simulations of parahydrogen in carbon nanotubes. ${ }^{31}$ The layering of the ${ }^{4} \mathrm{He}$ atoms has been attributed to the interplay between the attractive nature of the $\mathrm{He} / \mathrm{CNT}$ interaction and the hardcore of the $\mathrm{He}-\mathrm{He}$ interatomic potential. ${ }^{21,29}$ The He-DFT approach includes a density functional phenomenological term to characterize the short-range $\mathrm{He}-\mathrm{He}$ correlations arising from the $\mathrm{He}-\mathrm{He}$ potential wall, which has to be truncated at short distances. As the next step forward, we present benchmark nuclear wave function-based (NWF) results for $\left({ }^{4} \mathrm{He}\right)_{\mathrm{N}}$ clusters $(\mathrm{N}<4)$ inside CNTs of different radii. This method consists in explicitly solving the nuclear Schrödinger equation in the real space. For the discretization, we have used the Discrete Variable Representation (DVR) method. ${ }^{32}$ Within the selected model (adiabatic formulation, reduced dimension space for $\mathrm{N}_{2}$, and pairwise interaction approximation), this provides an exact solution if convergence with the DVR basis is achieved. Appealing advantages of the NWF method are the absence of the phenomenological terms, the employment of the bare (untruncated) $\mathrm{He}-\mathrm{He}$ potential, the inclusion of bosonic symmetry, and the possibility of calculating excited states. Our results are compared to those obtained for $\left(\mathrm{N}_{2}\right)_{N}(N<3)$, providing further insights into the experimental observation of Ohba. ${ }^{9}$ For the sake of consistency, the He-DFT approach has been applied as in Paper $\mathrm{I}^{21}$ but using the potential model proposed in this work. Moreover, we have developed a mixed NWF/He-DFT approach to get further insights into helium adsorption. Clearly, the considered Hamiltonian for the nuclear motion is a model, even within the adiabatic approximation. This Hamiltonian model does not include many-body terms for both (adsorbate cluster)-CNT and internal adsorbate interactions, and in the case of the $\mathrm{N}_{2}$ molecule, the internal degrees of freedom. These are, however, the common approximations used in all methods applied to this problem. The rotational motion for molecular adsorbates can be explicitly included into the NWF treatment, which will be the subject of a follow-up study.

The structure of this paper is as follows: In Section 2 the nuclear wave function-based method and the mixed NWF/HeDFT approach are presented. Section 3 focuses on the SAPTbased decomposition of the $\mathrm{He} / \mathrm{CNT}$ and $\mathrm{N}_{2} / \mathrm{CNT}$ interaction and discusses our new pairwise potential model. Section 4 analyzes the nuclear wave functions of $\left({ }^{4} \mathrm{He}\right)_{N}$ and $\left(\mathrm{N}_{2}\right)_{N}$ clusters inside and outside CNTs. The results from He-DFT and mixed He-DFT/NWF calculations are discussed in Section 5. Finally, Section 6 presents a global analysis of this work in the scenario of the experimental measurements and closes with the concluding remarks and future prospects. 


\section{THEORY}

2.1. Nuclear Wave-Function Approach: Hamiltonian Model. We have calculated the nuclear wave function of $N=$ $1-3{ }^{4} \mathrm{He}$ atoms or $1-2 \mathrm{~N}_{2}$ molecules in carbon nanotubes of helicity indices $(5,5)$ and $(9,9)$, with diameters below and above $0.7 \mathrm{~nm}$, respectively. The carbon atoms of the nanotube were kept frozen, choosing the experimental $\mathrm{C}-\mathrm{C}$ bond length of graphite $(1.42 \AA)$ for the nanotube geometries. Figure 1 illustrates the structural models of our systems.

We briefly outline the nuclear wave function-based (NWF) approach while the details are provided in the Supporting Information. To unify the notation, the adsorbate (the ${ }^{4} \mathrm{He}$ atom or the $\mathrm{N}_{2}$ molecule) is referred to as " $A$ " in the equations below. For the $\left(\mathrm{N}_{2}\right)_{\mathrm{N}} / \mathrm{CNT}$ system, as shown in Figure 1, the $\mathrm{N}_{2}$ molecules are described as structureless bosonic particles, with the $\mathrm{N}-\mathrm{N}$ bond oriented along the tube transversal axis $z$, i.e. with the $\left(\mathrm{N}_{2}\right)_{N}$ oligomeres in a parallel in-line configuration. Using cylindric coordinates $\mathbf{r}_{i} \equiv\left(r_{i}, \phi_{i}, z_{i}\right)$ for a reference ${ }^{4} \mathrm{He}$ atom, or the center-of-mass of a reference $\mathrm{N}_{2}$ molecule, contained in (or adsorbed to) a carbon nanotube, we assume the $Z$ axis of the body-fixed (BF) coordinate system parallel to the tube long axis $z$ (see Figure 1). Then, the one-particle Hamiltonian reads

$$
\hat{h}^{(i)}=-\frac{1}{2 M}\left(\frac{\partial^{2}}{\partial r_{i}^{2}}+\frac{1}{r_{i}^{2}} \frac{\partial^{2}}{\partial \phi_{i}^{2}}+\frac{\partial^{2}}{\partial z_{i}^{2}}\right)+V^{\mathrm{A}-\mathrm{CNT}}\left(r_{i}, \phi_{i}, z_{i}\right)
$$

The first term on the right-hand side is the kinetic energy operator expressed in cylindric coordinates with $M$ denoting the mass of the adsorbate. The second term on the left-hand side is the kinetic energy operator (referred to as $\hat{K}$ in the following), and the last term, $V^{\mathrm{A}-\mathrm{CNT}}$, is the adsorbate/CNT interaction potential. The total Hamiltonian of $N^{4} \mathrm{He}$ atoms or $\mathrm{N} \mathrm{N}_{2}$ molecules in the nanotube can be then written as

$$
\begin{aligned}
\hat{H} & =\sum_{i=1}^{N} K\left(\rho_{i}, \phi_{i}, z_{i}\right)+\sum_{i=1}^{N} V^{\mathrm{A}-\mathrm{CNT}}\left(r_{i}, \phi_{i}, z_{i}\right) \\
& +\sum_{i>j}^{N} V_{\mathrm{A}-\mathrm{A}}\left(R_{i j}\right)
\end{aligned}
$$

where $V_{\mathrm{A}-\mathrm{A}}$ is the interadsorbate $\left(\mathrm{He}-\mathrm{He}\right.$ or $\left.\mathrm{N}_{2}-\mathrm{N}_{2}\right)$ potential, with

$$
R_{i j}^{2}=r_{i}^{2}+r_{j}^{2}-2 r_{i} r_{j} \cos \left(\phi_{i}-\phi_{j}\right)+\left(z_{i}-z_{j}\right)^{2}
$$

The variable $R_{i j}$ corresponds to the distance between two $\mathrm{He}$ atoms or $\mathrm{N}_{2}$ molecules.

To preserve the cylindric symmetry of the system, the very small corrugation appearing along the azimuthal degree of freedom $\phi$ is ignored. Moreover (see also Paper $\mathrm{I}^{21}$ ), the adsorbate-CNT interaction is almost constant along the $z$ axis, decaying slowly at the borders. Then, considering long nanotubes, we can assume that the interaction potential depends only on $r$ (one-dimensional 1D model) and $V^{\mathrm{A}-\mathrm{CNT}}(r)$. Using this special form of the interaction potential, the $z$ components of the total angular momentum, $\Lambda$, and the total momentum, $P_{Z}$, are conserved. Therefore, the coordinates corresponding to the global rotation about $z, \Phi=\sum_{i} \phi_{i} / N$, and the collective motion along $z, Z=\sum_{i} z_{i} / N$, can be separated. Explicitly, the bound-state wave functions for a single ${ }^{4} \mathrm{He}$ atom or $\mathrm{N}_{2}$ molecule in the nanotube can be written as (for $Z=0$ )

$$
\psi_{m}^{n}(r, \phi)=\frac{\exp (\operatorname{lm} \phi)}{\sqrt{2 \pi}} \psi_{m}^{n}(r)
$$

which introduces the quantum number $m$ as the $z$ component of the total angular momentum. The single-particle bound states $\psi_{m}^{n}(r)$ (referred to as nuclear orbitals) are calculated by solving numerically the Schrödinger equation $\hat{h}_{m} \psi_{m}^{n}(\mathbf{r})=$ $\epsilon_{m}^{n} \psi_{m}(\mathbf{r})$, with (see Supporting Information)

$$
\hat{h}_{m}=-\frac{1}{2 M}\left(\frac{\partial^{2}}{\partial r^{2}}-\frac{m^{2}-1 / 4}{r^{2}}\right)+V^{\mathrm{A}-\mathrm{CNT}}(r)
$$

To this end, we used 2D-harmonic oscillator functions of a frequency $w$ depending on the adsorbate $\left({ }^{4} \mathrm{He}\right.$ or $\left.\mathrm{N}_{2}\right)$ and the adsorbate location (inside or outside of the carbon nanotube). These nuclear orbitals served as a potential-optimized basis set to calculate the nuclear wave function for $N=2$ and 3 . For the case of $N=2$, after separating the overall rotation $\Phi$ and the overall displacement $Z$, the total wave function can be written as

$$
\begin{aligned}
& \Psi_{\Lambda}^{n}\left(r_{1}, r_{2}, \phi_{12}, z_{12}, \Phi, Z\right) \\
& \quad=\frac{\exp (\iota \Lambda \Phi)}{\sqrt{2 \pi}} \exp (\iota K Z) \Psi_{\Lambda}^{n}\left(r_{1}, r_{2}, \phi_{12}, z_{12}\right)
\end{aligned}
$$

with $\phi_{12}=\phi_{2}-\phi_{1}$, and $z_{12}=z_{2}-z_{1}$. Once again, $K$ represents the overall $Z$ momentum and can be omitted $(K=0)$. The remaining four-dimensional $4 \mathrm{D}$ wave function of bosonic ${ }^{4} \mathrm{He}$ atoms or $\mathrm{N}_{2}$ molecules must be symmetric,

$$
\Psi_{\Lambda}^{n}\left(r_{2}, r_{1},-\phi_{12},-z_{12}\right)=\Psi_{\Lambda}^{n}\left(r_{1}, r_{2}, \phi_{12}, z_{12}\right)
$$

The $\Psi_{\Lambda}^{n}$ wave function is a solution of the ( $\Lambda$-dependent) $4 \mathrm{D}$ Hamiltonian,

$$
\hat{H}_{\Lambda}=\hat{K}_{\Lambda}+V^{\mathrm{A}-\mathrm{CNT}}\left(r_{1}\right)+V^{\mathrm{A}-\mathrm{CNT}}\left(r_{2}\right)+V_{\mathrm{A}-\mathrm{A}}\left(R_{12}\right)
$$

with the explicit form of $\hat{K}_{\Lambda}$ given by

$$
\begin{aligned}
\hat{K}_{\Lambda}= & -\frac{1}{2 M}\left\{\frac{\partial^{2}}{\partial r_{1}^{2}}+\frac{1}{r_{1}} \frac{\partial}{\partial r_{1}}+\frac{\partial^{2}}{\partial r_{2}^{2}}+\frac{1}{r_{2}} \frac{\partial}{\partial r_{2}}+2 \frac{\partial^{2}}{\partial z_{12}^{2}}\right. \\
& \left.+\left(\frac{1}{r_{1}^{2}}+\frac{1}{r_{2}^{2}}\right)\left(\frac{\partial^{2}}{\partial \phi_{12}^{2}}-\Lambda^{2}\right)+\imath \Lambda\left(\frac{1}{r_{2}^{2}}-\frac{1}{r_{1}^{2}}\right) \frac{\partial}{\partial \phi_{12}}\right\}
\end{aligned}
$$

The 4D Hamiltonian problem is solved by using the Discrete Variable Representation (DVR) approach. ${ }^{32}$ The basis set is obtained as a direct product of functions for the $r_{i}, z_{12}$, and $\phi_{12}$ coordinates. For the $r_{i}$ degrees of freedom, we use the potentialoptimized DVR functions ${ }^{33}$ corresponding to the solution of eq 3 , built from $2 \mathrm{D}$-harmonic oscillator functions. For $z_{12}$ and $\phi_{12}$ coordinates, Sinc-DVR functions are employed, with the latter corresponding to $\exp \left(\operatorname{lm} \phi_{12}\right)$ harmonics. A similar approach is applied for $N=3$, where the Hamiltonian becomes seven dimensional (7D); see the Supporting Information for further details.

2.2. Mixed Nuclear Wave-Function and Helium Density Functional Approach. To study larger clusters accounting for quantum effects, we combine NWF and Helium Density Functional (He-DFT) approaches. As in Paper I, ${ }^{21}$ the system consists of a ${ }^{4} \mathrm{He}_{2000}$ droplet containing carbon nanotubes of several size. We assume that the helium droplet is a Bose-Einstein condensate with all the ${ }^{4} \mathrm{He}$ atoms in the same single-particle state. Then, it can be represented by an effective macroscopic wave function $\Psi(\mathbf{r})$ so that the density is 

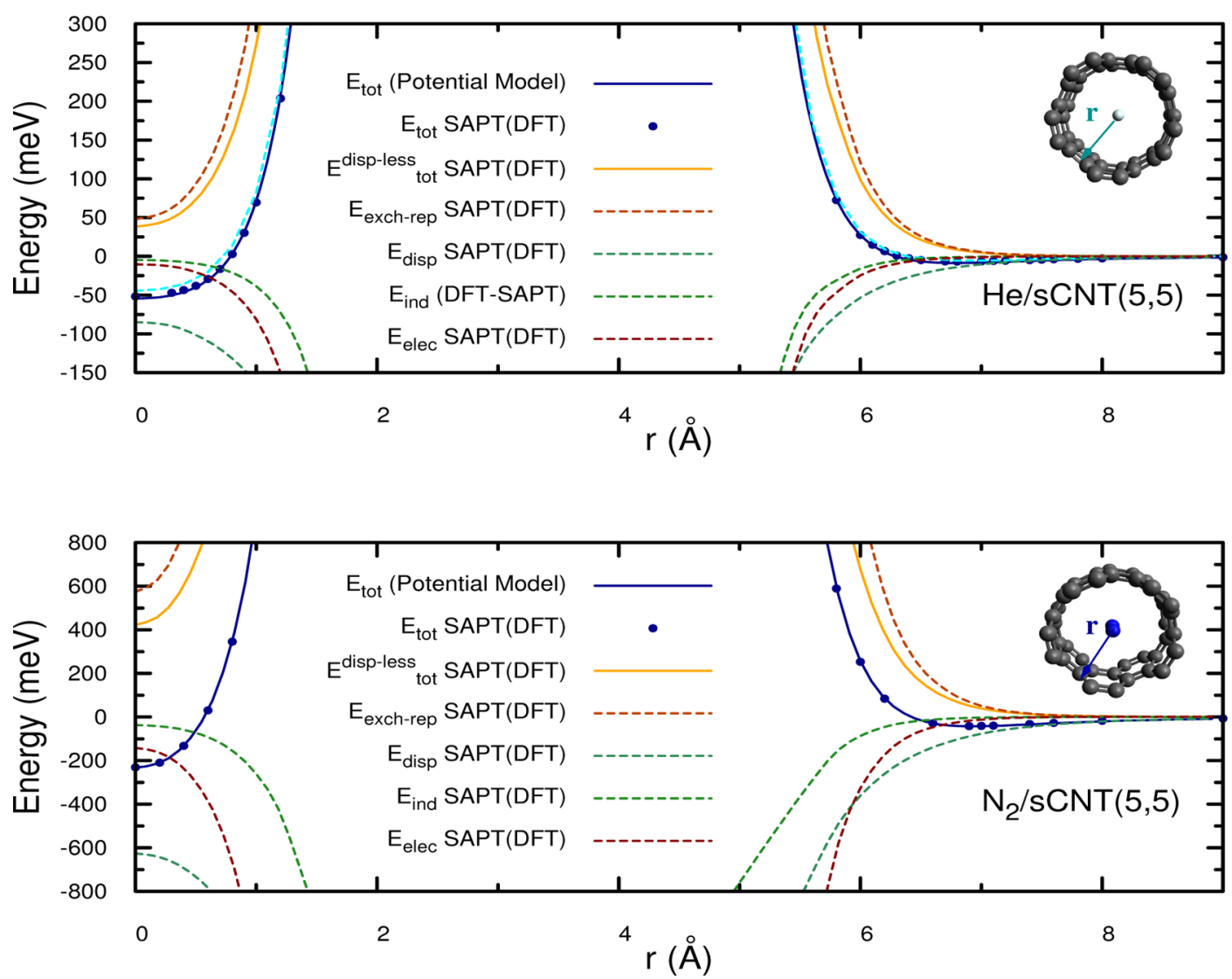

Figure 2. SAPT(DFT)-based decomposition of the interaction energies between a single He atom (upper panel) and a $\mathrm{N}_{2}$ molecule (lower panel) with a short carbon nanotube $(\mathrm{sCNT})$ of helicity index $(5,5)$ and a radius of $3.37 \AA$. The He atom and the $\mathrm{N}_{2}$ mass center are located at a distance $r$ from the tube mass center, at the central plane perpendicular to the tube direction $(z)$, with the $\mathrm{N}-\mathrm{N}$ bond oriented along the same $z$ axis. The cyan line (upper panel) corresponds to SAPT(DFT) energies calculated with the cc-pVTZ basis for carbon atoms. ${ }^{21}$

defined as $\rho(\mathbf{r})=|\Psi(\mathbf{r})|^{2}$. We minimize the free energy $F[\rho]$, a functional of the helium density $\rho$,

$$
F[\rho]=E[\rho]+U_{\mathrm{He}-\mathrm{CNT}}[\rho]-\mu N[\rho]-\mathbf{F} \cdot \mathbf{R}[\rho]
$$

with $E[\rho]$ denoting a slightly modified version of the OrsayTrento-density functional, ${ }^{27,34} \mu$ and $\mathbf{F}$ being the chemical potential and the retaining force, and $U_{\mathrm{He}-\mathrm{CNT}}[\rho]$ introducing the droplet/nanotube interaction:

$$
U_{\mathrm{He}-\mathrm{CNT}}[\rho]=\int \mathrm{d} \mathbf{r} \rho(\mathbf{r}) V^{\mathrm{He}-\mathrm{CNT}}(\mathbf{r})
$$

where $V^{\mathrm{He}-\mathrm{CNT}}$ is the $\mathrm{He} / \mathrm{CNT}$ interaction potential (unlike Paper $\mathrm{I}^{21}$ our new $\mathrm{He} / \mathrm{CNT}$ potential model has been employed here). The last two terms entering eq 4 allow for the conservation of $N$, the particle number, $N=\int \mathrm{d} \mathbf{r} \rho(\mathbf{r})$, and $\mathbf{R}$, the He droplet mass center, $\mathbf{R}=1 / N \int \mathrm{d} \mathbf{r} \rho(\mathbf{r}) \mathbf{r}$. The density functional $E[\rho]$ reads $^{34}$

$$
\begin{aligned}
E[\rho]= & \frac{\hbar^{2}}{2 M} \int \mathrm{d} \mathbf{r}(\nabla \sqrt{\rho}(\mathbf{r}))^{2} \\
& +\frac{1}{2} \int \mathrm{d} \mathbf{r} \mathrm{d} \mathbf{r}^{\prime} \rho(\mathbf{r}) V_{\mathrm{He}-\mathrm{He}}\left(\left|\mathbf{r}-\mathbf{r}^{\prime}\right|\right) \rho\left(\mathbf{r}^{\prime}\right) \\
& +\frac{c_{2}}{2} \int \mathrm{d} \mathbf{r} \rho(\mathbf{r}) \bar{\rho}(\mathbf{r})^{2}+\frac{c_{3}}{2} \int \mathrm{d} \mathbf{r} \rho(\mathbf{r}) \bar{\rho}(\mathbf{r})^{3} \\
& +C \int \mathrm{d} \mathbf{r}\left[1+\tanh \left(\beta\left\{\rho(\mathbf{r})-\rho_{m}\right\}\right)\right]
\end{aligned}
$$

where the right-hand terms can be identified as the quantum kinetic energy, the mean-field $\mathrm{He}-\mathrm{He}$ interaction via a truncated $\mathrm{He}-\mathrm{He}$ potential, and two terms accounting phenomenologically for short-range correlations involving $\bar{\rho}$, a locally averaged density for a given sphere of radius $\bar{h}$. For these short-range correlation energy contributions, we chose the original parameters $\bar{h}, c_{2}$, and $c_{3}$ of the Orsay-Trento density functional, reproducing the experimental density, energy per atom, and compressibility for the liquid at zero temperature and pressure. ${ }^{27}$ The last term in eq 6 is a penalty term avoiding unphysical piling-up of He density with $\rho_{m}$ as threshold value. For details we refer to refs 34 and 35 .

As a second step, the total density of the ${ }^{4} \mathrm{He}_{2000}$ droplet, $\rho(\mathbf{r})$, is partitioned into the innermost helium layer, $\rho_{\mathrm{I}}(\mathbf{r})$, and the second and all following layers of helium, $\rho_{\mathrm{II}}(\mathbf{r})$. Next, we calculate the nuclear wave function of a single ${ }^{4} \mathrm{He}$ atom located in region I. This is where both conceptually different approaches meet: The innermost layer of helium (i.e., region I) is now replaced by a real $\mathrm{He}$ atom, switching from a continuous into a discrete picture, while the remaining $\mathrm{He}$ density in region II is still treated at the He-DFT level of theory. The total potential felt by the ${ }^{4} \mathrm{He}$ atom in region I can be approximated as the sum of the $\mathrm{He} / \mathrm{CNT}$ potential and an embedding potential arising from the ${ }^{4} \mathrm{He}$ atoms located in region II, $V_{\text {tot }}(\mathbf{r})=V^{\mathrm{He}-\mathrm{CNT}}(\mathbf{r})+V_{\mathrm{emb}}(\mathbf{r})$. Specifically, the embedding potential is defined as

$$
V_{\mathrm{emb}}(\mathbf{r})=\int \mathrm{d} \mathbf{r}^{\prime} V_{\mathrm{He}-\mathrm{He}}\left(\left|\mathbf{r}-\mathbf{r}^{\prime}\right|\right) \rho_{\mathrm{II}}\left(\mathbf{r}^{\prime}\right)
$$


Table 1. SAPT-Based Decomposition of the $\mathrm{He} / \mathrm{sCNT}(5,5)$ and $\mathrm{N}_{2} / \mathrm{sCNT}(5,5)$ Interaction Energies Using the SAPT(DFT) Method $^{a}$

$\begin{array}{lcccc} & \mathrm{He} / \mathrm{sCNT}(5,5) \text { inside } & \mathrm{He} / \mathrm{sCNT}(5,5) \text { outside } & \mathrm{N}_{2} / \mathrm{sCNT}(5,5) \text { inside } & \mathrm{N}_{2} / \mathrm{sCNT}(5,5) \text { outside } \\ r_{e} \AA & 0.0 & 6.8 & 0.0 & 7.0 \\ E_{\text {elec }}, \mathrm{meV} & -10.27 & -1.99 & -142.09 & -12.27 \\ E_{\text {exch-rep, }} \mathrm{meV} & 48.13 & 10.29 & 574.30 & 51.05 \\ E_{\text {ind }} \mathrm{meV} & -4.64 & -1.02 & -37.45 & -3.08 \\ E_{\text {disp }}, \mathrm{meV} & -84.96 & -14.08 & -626.09 & -76.97 \\ E_{\text {tot }} \mathrm{meV} & -51.75 & -6.80 & -231.33 & -41.28 \\ \delta(\mathrm{HF}), \mathrm{meV} & -0.18 & -0.04 & -4.93 & -0.62\end{array}$

${ }^{a}$ Both adsorbates are located at the potential minima positions $\left(r_{e}\right)$ inside and outside the nanotube cage (see Figure 1).

preserving the cylindric symmetry of the system. The nuclear wave function of the embedded ${ }^{4} \mathrm{He}$ atom can be written as in eq 2 but considering two-dimensional $\psi_{m}^{n}(r, z)$ functions to account for border effects in the finite length nanotubes. These functions are obtained using the DVR approach, as a product of 2D-HO functions for the coordinate $r$ and Sinc-DVR functions for the $z$ variable.

\section{RESULTS AND DISCUSSION: HE/NANOTUBE AND $\mathrm{N}_{2}$ /NANOTUBE INTERACTION POTENTIALS}

3.1. SAPT(DFT) Interaction Energies. Let us now focus on the potential felt by a single $\mathrm{He}$ atom or a $\mathrm{N}_{2}$ molecule inside and outside of a carbon nanotube. Considering an unsaturated $\operatorname{CNT}(5,5)$ tube made of 40 carbon atoms, we apply the SAPT(DFT) method as implemented within MOLPRO. ${ }^{36,37}$ The $\operatorname{CNT}(5,5)$ is chosen because its diameter is below the threshold value $(0.7 \mathrm{~nm})$ for which the experimental measurements indicate a very limited helium adsorption. ' We followed the same computational setup detailed in Paper $\mathrm{I}^{21}$ using the Perdew-Burke-Ernzerhof (PBE) density functional, ${ }^{38}$ but enlarge the electronic basis set to a modified version of the augmented polarized correlationconsistent triple $-\zeta$ basis $^{39}$ (aug-cc-pVTZ) for the nanotube carbon atoms as well as for the helium and nitrogen atoms. To solve linear dependence problems, the carbon basis was modified by multiplying the most diffuse exponents for all angular momenta by a factor of 2.3. This approach was carefully validated in previous studies on the carbon nanostructures. ${ }^{24,40}$ Applying the density-fitting (DF) technique, these calculations become feasible. $^{36,37}$ The DF of Coulomb and exchange integrals has used the auxiliary basis set developed for the augcc-pVTZ basis by Weigend, ${ }^{41}$ while the aug-cc-pVTZ/MP2Fit basis $^{42}$ was employed to fit the integrals containing virtual orbitals. The exchange-correlation PBE potential is asymptotically corrected ${ }^{43}$ using the ionization potential (IP) value reported in the NIST Chemistry Web Book for the He atom ${ }^{44}$ while the nanotube IP values are estimated using the DFT PBE0 approach ${ }^{45}$ instead. We note that for the considered nanotubes the saturation of the dangling bonds at the tube borders showed interaction energy differences below $6 \%$ at the potential minima.

The SAPT(DFT) method decomposes the interaction energy in first-order electrostatic $E_{\text {elec }}$ and exchange-repulsion terms $\mathrm{E}_{\text {exch }}$ second-order induction $E_{\text {ind }}$ and dispersion $E_{\text {disp }}$ terms, along with their respective exchange corrections $\left(E_{\text {exch-ind }}\right.$ and $\left.E_{\text {exch-disp }}\right)$. The $\delta(\mathrm{HF})$ estimates ${ }^{37,46}$ of induction contributions beyond second-order terms are also calculated. We combine the dispersion and exchange-dispersion contributions into the total dispersion portion, as the induction, exchange-induction, and $\delta(\mathrm{HF})$ contributions are brought together into the total induction term.

Figure 2 shows the energy decomposition as a function of the radial distance $r$ between the $\mathrm{He}$ atom (upper panel) or the $\mathrm{N}_{2}$ center-of-mass (bottom panel) and the nanotube center. We collect these contributions at the corresponding potential minima in Table 1 . For the sake of clarity, the electrostatic $E_{\text {elec }}$ exchange-repulsion $E_{\text {exch-rep, }}$ and induction $E_{\text {ind }}$ terms are summed to provide the total dispersionless interaction (referred to as $E_{\text {tot }}^{\text {disp-less }}$ ). A comparison between the additive pairwise potential model and SAPT(DFT) potential energy curves is also shown (see next subsection). The upper panel of Figure 2 also incorporates SAPT(DFT) interaction energies obtained with the smaller cc-pVTZ basis on carbon atoms. ${ }^{21}$ The enlargement of the basis set from cc-pVTZ to aug-ccpVTZ raises the attractive interaction by up to $14 \%$ and $18 \%$ for $\mathrm{He}$ and $\mathrm{N}_{2}$, respectively.

Figure 2 and Table 1 clearly show that the adsorbate/ nanotube interactions are dominated by the dispersion at the potential minima, representing at least $78 \%$ of the attractive energy contribution. The dispersion also determines the global interaction at distances larger than $4.6 \AA$ from the carbon wall. Naturally, the larger number of electrons and the polarizability of the $\mathrm{N}_{2}$ molecule (about $11.77^{47}$ vs 1.38 au for $\mathrm{He}^{48}$ ) translate into more attractive dispersion terms, causing an increase by a factor of 7 at the global minima ( -626 vs -85 $\mathrm{meV}$ ). Obviously, the larger number of electrons of the $\mathrm{N}_{2}$ molecule leads to a large overlap with carbon densities and a more marked exchange-repulsion ( 574 vs $48 \mathrm{meV}$ ), which is however fully compensated by the more attractive dispersion term and electrostatic component $(-142$ vs $-10 \mathrm{meV})$, as expected from the quadrupole-quadrupole interaction for $\mathrm{N}_{2}$ and its absence for helium. As a result, the $\mathrm{N}_{2} / \mathrm{CNT}$ well is about a factor of 4.5 deeper. For both adsorbates the induction contribution is only significant at the potential barrier, accounting for $3-5 \%$ of the total interaction at the potential minima (see Table 1$)$. The $\delta(\mathrm{HF})$ corrections are very small, representing $4-20 \%$ of the total induction term at the potential minima. Unlike the dispersion (see Figure 2 and Table 1), the dispersionless interaction is overly repulsive, mostly determined by the exchange-repulsion, and grows exponentially as the adsorbate-wall distance decreases.

Another clear feature of Figure 2 is the asymmetry of the potential energy curve depending on the internal or external adsorbate location with respect to the $\operatorname{CNT}(5,5)$ carbon wall. As expected from the interaction with a larger number of $\mathrm{C}$ atoms, the inner potential minima are much deeper than outer ones. In contrast, the inner and outer potential minima positions (see Table 1) are almost symmetric with respect to the tube wall (about $3.4 \AA$ for $\mathrm{He}$ ). Notice that the relatively 

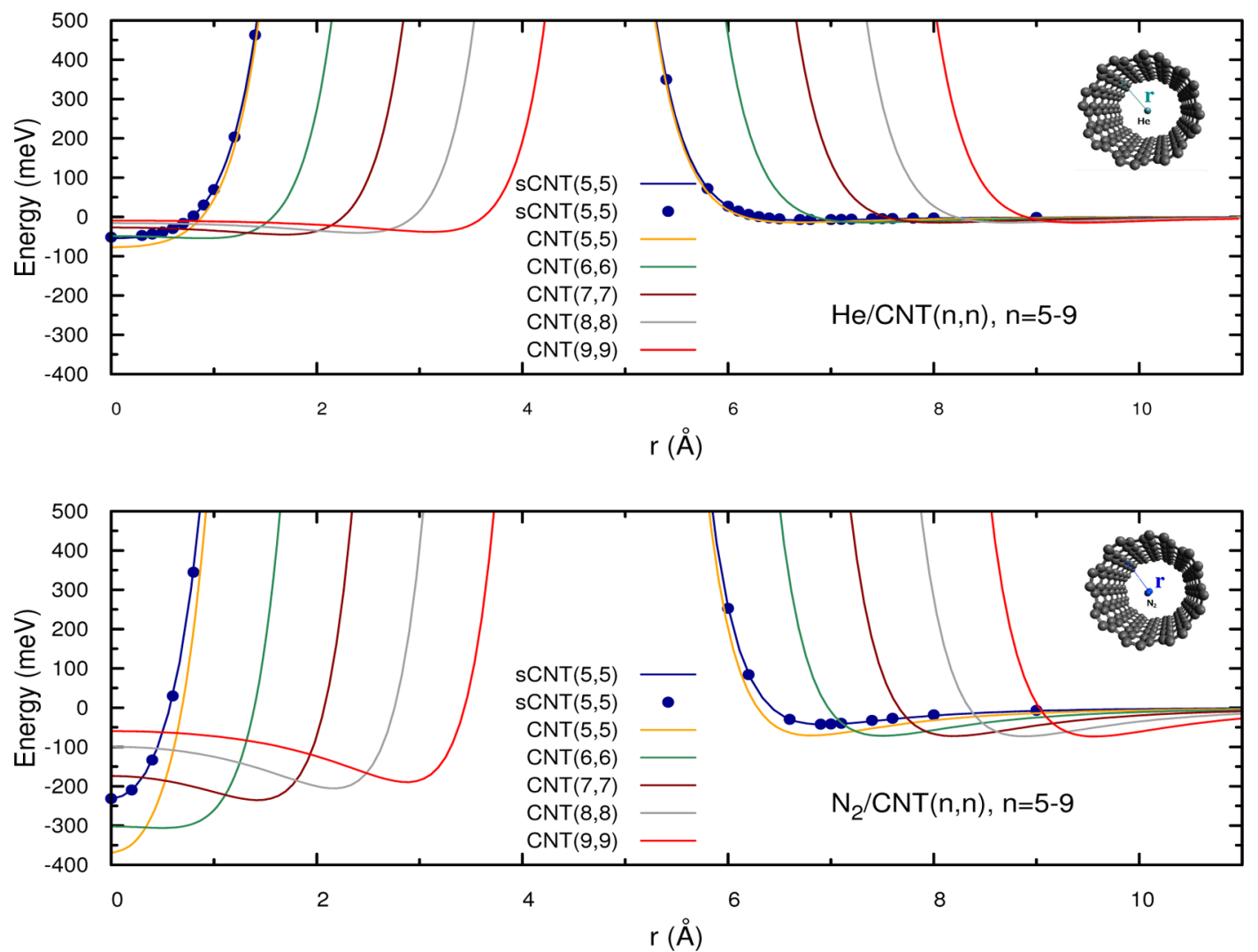

Figure 3. Radial scans of the PES for the interaction of a single He atom (upper panel) and a $\mathrm{N}_{2}$ molecule (lower panel) with carbon nanotubes (CNT) of various size, using our proposed pairwise potential model (PPM). PPM and SAPT(DFT) interaction energies are plotted in blue for the short carbon nanotube, abbreviated as $\operatorname{sCNT}(5,5)$, with a length of $3.69 \AA$.

short $\mathrm{CNT}(5,5)$ radius (about $3.4 \AA$ ) allows the adsorbates to benefit from the attractive dispersion interaction with the fluctuating dipoles located at $\mathrm{C}-\mathrm{C}$ bonds at opposite sides of the nanotube cage. The exchange-repulsion is also large at the nanotube center but completely quenched by the dispersion interaction. In fact, the exchange-repulsion decays much more rapidly (exponentially). Additionally, the carbon density is higher at the CNT inner wall, also contributing to enhance the interaction potential asymmetry. In this way, the $\mathrm{He} / \mathrm{CNT}$ exchange-repulsion at the tube center is not just twice that at the potential minima on the outside but almost five times larger (see Table 1).

3.2. Pairwise Potential Model. We have designed an additive pairwise potential model (PPM) to fit the dispersionless and dispersion SAPT(DFT) energy contributions. This model is a modified version of that previously developed for $\mathrm{He} / \mathrm{Mg}(0001)$ and $\mathrm{He} / \mathrm{C}_{60}$ interactions. ${ }^{19,22}$ It also extends the Lennard-Jones function developed by Carlos and Cole ${ }^{49,50}$ to model the adsorption of noble-gases onto the graphite surface, ${ }^{50}$ and later also on carbon nanotubes. ${ }^{51}$ The dispersion energies are fitted using the pairwise $D_{a s}$ functional of Szalewicz and collaborators, ${ }^{52-54}$ but modulated by an additional term characterizing the anisotropy in the pair adsorbate-carbon interaction: ${ }^{5}$

$$
\begin{aligned}
E_{\text {int }}^{\text {disp }}\left(\left\{\mathbf{R}_{\mathrm{HeC}}\right\}\right)= & -\sum_{\mathrm{C}}\left[1+\gamma_{A}\left(1-\frac{3}{2} \cos ^{2} \theta_{\mathrm{C}}\right)\right] \times \\
& \sum_{n=6,8} \frac{\sqrt{C_{n}^{\mathrm{He}} C_{n}^{\mathrm{C}}}}{R_{\mathrm{HeC}}^{n}} f_{n}\left(\sqrt{\beta_{\mathrm{He}} \beta_{\mathrm{C}}} R_{\mathrm{HeC}}\right)
\end{aligned}
$$

where $R_{\mathrm{HeC}}$ stands for the distance between the He atom (or $\mathrm{N}_{2}$ mass center) and one carbon atom of the nanotube, and $\theta_{\mathrm{C}}$ is the angle between the radial vector going from the nanotube center to one carbon atom and the vector $\mathbf{R}_{\mathrm{HeC}}$ pointing from the $\mathrm{He}$ atom to the same $\mathrm{C}$ atom. The dimensionless factor $\gamma_{A}$ in the first term is the parameter accounting for the anisotropy of the $\mathrm{C}-\mathrm{C}$ bonds. The sum in eq 8 runs over all carbon atoms of the nanotube, and the terms $f_{n}(n=6,8)$ stand for the damping functions of Tang and Toennies. ${ }^{55}$ Interestingly, our $\mathrm{ab}$ initio tuning provides $\gamma_{A}$ values $(0.4-0.6)$ of similar magnitude to those used in studies on the phase transition of noble gases in nanotubes ( 0.38 from ref 51).

As mentioned above, the dispersionless adsorbate/CNT energies are overly repulsive and scale exponentially as the distance between the interacting species decreases. They are well fitted by the function

$$
\begin{aligned}
& E_{\text {int }}^{\text {disp-less }}\left(\left\{\mathbf{R}_{\mathrm{HeC}}\right\}\right) \\
& \quad=\sum_{\mathrm{C}}\left[1+\gamma_{R}\left(1-\frac{6}{5} \cos ^{2} \theta_{\mathrm{C}}\right)\right] \times A e^{\left(-\alpha R_{\mathrm{HeC}}-\beta R_{\mathrm{HeC}}^{2}\right)}, R_{\mathrm{HeC}}<R_{c} \\
& =0, \quad R_{\mathrm{HeC}}>R_{c}
\end{aligned}
$$

with $R_{c}$ as cutoff distance. This function is well-defined for $R_{\mathrm{HeC}}$ $>1 \AA$. Once again, the dimensionless factor $\gamma_{R}$ is the anisotropy parameter. The values of the $\mathrm{He} / \mathrm{CNT}$ and $\mathrm{N}_{2} / \mathrm{CNT}$ parameters are provided as Supporting Information.

Figure 3 shows radial scans of the potential energy surfaces (PES) obtained by applying the PPM model to carbon nanotubes of various diameters (from $\sim 3.4$ to $12 \AA$ ) and a length of about $13.5 \AA$, along with the short $(5,5)$ nanotube, 
abbreviated as $\operatorname{sCNT}(5,5)$, considered in the $\operatorname{SAPT}(\mathrm{DFT})$ calculations (about $3.7 \AA$ long). Notice that the increase of the nanotube length makes the interaction more attractive (see Figure 3). A nanotube of shorter diameter results in a net repulsive interaction of either $\mathrm{He}$ or $\mathrm{N}_{2}$ with the internal carbon cage. The $\mathrm{He} / \mathrm{CNT}$ radial scans are similar to those presented using the atom-bond potential model in Paper $\mathrm{I}^{21}$ The improvement brought by our PPM model lies mainly in the better characterization of the hard-core of the interaction as well as the asymmetry of the potentials with respect to the carbon cage location. Our PPM model fits almost perfectly the SAPT(DFT) interaction energies both inside and outside the $\operatorname{sCNT}(5,5)$ nanotube. The same holds for the separate dispersionless and dispersion interactions. The model relies on two basic conclusions from accurate studies of van der Waals-dominated adsorbate/surface systems: ${ }^{14-19}$ (1) the short-range nature of the dispersionless contribution allows an appropriate tuning of model parameters using ab initio energies on small surface cluster models; (2) the dispersion is long-range, but dispersion parameters show excellent transferability properties upon increasing the size of the surface cluster models. ${ }^{18}$ The first outcome is reflected in the very similar potential wall for the short and long $\operatorname{CNT}(5,5)$ nanotubes (see Figure 3).

Notice that the dependence of the interaction energies in Figure 3 on the tube radius is qualitatively very similar for $\mathrm{He}$ and $\mathrm{N}_{2}$. In the outer region, the increase of the nanotube radius results in the shifting of the potential minima position to larger $r$ values, with well-depths varying very slowly and converging to the values obtained for a planar graphene sheet. The variation of the inner potential landscape with the tube radius is more pronounced: the larger the nanotube radius, the smaller the dispersion is at the tube center. As a consequence, the potential minimum moves from the center to positions closer to the carbon wall. Also, the decrease of the cage curvature with increasing tube radius makes the carbon density at the inner surface more diffuse, and the adsorbate/nanotube exchangerepulsion weaker. As a result, the distance from the inner carbon wall to the adsorbate shrinks down to $0.5 \AA$ (see Table 2).

Table 2. Decomposition of $\mathrm{He} / \mathrm{CNT}$ and $\mathrm{N}_{2} / \mathrm{CNT}$ Interaction Energies in Dispersionless and Dispersion Contributions $^{a}$

$\begin{array}{lcccc} & \begin{array}{c}\mathrm{He} / \\ \mathrm{CNT}(5,5) \\ \text { inside }\end{array} & \begin{array}{c}\mathrm{He} / \\ \mathrm{CNT}(9,9) \\ \text { inside }\end{array} & \begin{array}{c}\mathrm{N}_{2} / \\ \mathrm{CNT}(5,5) \\ \text { inside }\end{array} & \begin{array}{c}\mathrm{N}_{2} / \\ \mathrm{CNT}(9,9) \\ \text { inside }\end{array} \\ r_{\mathrm{CNT}}, \AA & 3.4 & 6.1 & 3.4 & 6.1 \\ r_{e} \AA & 0.0 & 3.2 & 0.0 & 3.0 \\ E_{\text {disp-less }}, \mathrm{meV} & 30.99 & 27.83 & 398.77 & 166.90 \\ E_{\text {disp }}, \mathrm{meV} & -108.10 & -65.31 & -765.63 & -351.57 \\ E_{\text {tot }}, \mathrm{meV} & -77.11 & -37.48 & -366.66 & -184.67\end{array}$

${ }^{a}$ Both adsorbates are located at the potential minima $\left(r_{e}\right)$ inside the nanotube. The CNT radius $\left(r_{\mathrm{CNT}}\right)$ is also indicated.

\section{RESULTS AND DISCUSSION: NUCLEAR BOUND STATES OF $\left({ }^{4} \mathrm{HE}\right)_{N} / C N T$ AND $\left(\mathrm{N}_{2}\right)_{N} / C N T$ SYSTEMS}

4.1. Nuclear Bound States of ${ }^{4} \mathrm{He} / \mathrm{CNT}$ and $\mathrm{N}_{2} / \mathrm{CNT}$ Complexes. Let us now compare the He-CNT and $\mathrm{N}_{2}-\mathrm{CNT}$ nuclear motions in carbon nanotubes: Figure 4 presents the radial densities of all bound states with $m=0$ while Table 3 collects the energies $\left(\epsilon_{m}^{n}\right.$, in $\left.\mathrm{meV}\right)$ of bound states for $m<6$, considering three representative cases only. The first thing to notice is that the oscillation amplitude for the radial He-CNT motion is more than twice larger than for the $\mathrm{N}_{2}-\mathrm{CNT}$ counterpart. Also, the much deeper $\mathrm{N}_{2} / \mathrm{CNT}$ potential supports a larger number of bound states for both $m=0$ (see Figure 4) and $m>0$ (see Table 3) and they are closer in energy to each other.

Due to the very pronounced repulsive hard-core at the carbon wall, we can separate the bound states for the adsorbate inside and outside the nanotube. Notice from Figure 4 that there are only three vibrational states with $m=0$ upon ${ }^{4} \mathrm{He}$ atom adsorption on the inner $\operatorname{CNT}(5,5)$ surface. Since the potential minimum is located at $r_{e}=0$ (see Table 2), the rotational constant for azimuthal ${ }^{4} \mathrm{He}$ motion is rather large (see eq 3) so that the energy of states with $m>0$ differs significantly from those with $m=0$ (see Table 3 ). Inside the tube, the total number of bound states for the ${ }^{4} \mathrm{He} / \mathrm{CNT}(5,5)$ complex is just ten, which is an order of magnitude smaller than that for the $\mathrm{N}_{2}$ counterpart. For the temperature of the experiment $^{9}(T=2-5 \mathrm{~K})$, however, only the lowest bound state holds a significant population in both ${ }^{4} \mathrm{He}$ and $\mathrm{N}_{2}$ cases. As typically found in classical descriptions of molecular adsorption in nanotubes, upon increasing the temperature, the $\mathrm{N}_{2}$ molecule tends to move along the attractive tail of the interaction potential, as close as possible to the carbon wall, as can be seen from the position of the maximum amplitude of the wave function (see Figure 4).

When the ${ }^{4} \mathrm{He}$ atom adsorbs inside the $\operatorname{CNT}(9,9)$ nanotube, the shifting of the potential minimum from the center of the nanotube to the lateral region $\left(r_{e}=3.2 \AA\right)$ causes the dropping of the rotational constant to very small values so that each vibrational solution with $m=0$ is followed by a tail of rotational states with $m>0$ (see Table 3 ). The number of bound states is thus a factor of 8 larger for ${ }^{4} \mathrm{He} / \mathrm{CNT}(9,9)$ than for ${ }^{4} \mathrm{He} /$ $\mathrm{CNT}(5,5)$. Assuming a Boltzmann distribution at $T=5 \mathrm{~K}$, only the lowest bound state of the ${ }^{4} \mathrm{He} / \mathrm{CNT}(5,5)$ complex is populated while six states become populated for the ${ }^{4} \mathrm{He} /$ $\mathrm{CNT}(9,9)$ system. As for a ${ }^{4} \mathrm{He}$ atom adsorbed outside the nanotubes, notice from Figure 4 that the very shallow interaction potentials support just five bound states characterized by very wide amplitudes (beyond $1.0 \AA \AA$ ). Upon increasing the $r_{e}$ value, the rotational constant decreases so that each vibrational state has associated its own rotational tail. The enlargement of the nanotube radius affects very slightly the interaction potentials and then the vibrational levels. These levels lie very close to those experimentally determined for a single ${ }^{4} \mathrm{He}$ atom adsorbed onto the graphite surface. ${ }^{56}$

4.2. Nuclear Bound States of $\left({ }^{4} \mathrm{He}\right)_{N} / \mathrm{CNT}(n, n)$ Complexes $(N>1)$. Let us now focus on how the inclusion of the $\mathrm{He}-\mathrm{He}$ interaction influences the nuclear bound states of $\left({ }^{4} \mathrm{He}\right)_{N} / \mathrm{CNT}(n, n)$ complexes $(N \leq 3)$. We have employed the very accurate $\mathrm{He}-\mathrm{He}$ potential of Cencek at al., ${ }^{10}$ which includes nonadiabatic and relativistic corrections. As explained in the methodological section, the total wave function depends on four and seven variables for $N=2$ and 3 , respectively, and it is expressed as a sum of direct products of basis functions for each coordinate. Specifically, 2D-harmonic oscillator functions are used for the radial $r_{i}$ variables while Sinc-DVR functions are employed for the relative distance along $z$ and angular, relative angles, and degrees of freedom; see the Supporting Information for further details. 

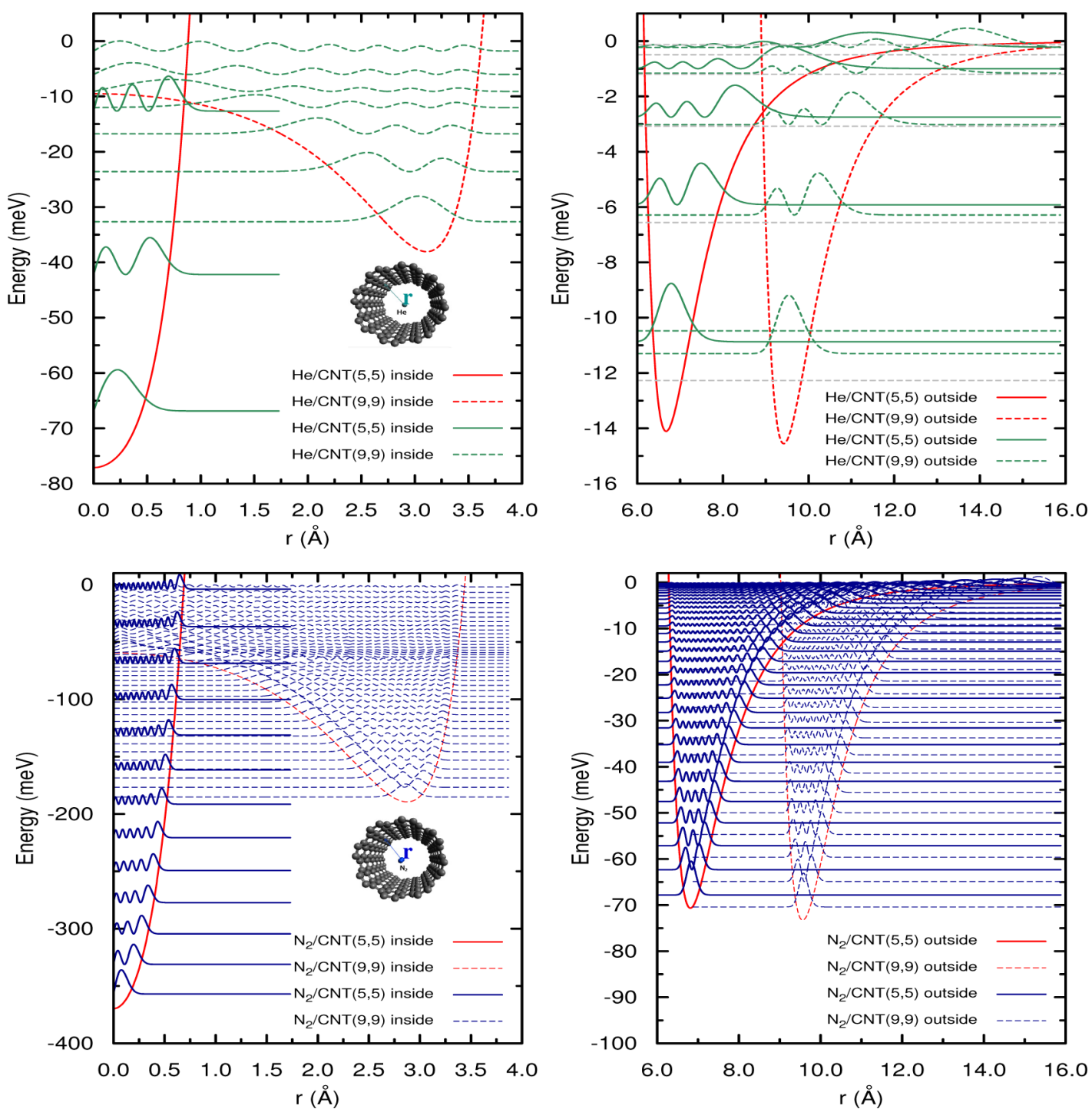

Figure 4. Radial densities $D(r)$ (in $\left.\AA^{-1}\right)$ corresponding to the nuclear bound states supported by $\left({ }^{4} \mathrm{He}\right) / \mathrm{CNT}(5,5)$ and $\left(\mathrm{N}_{2}\right) / \mathrm{CNT}(9,9)$ interaction potentials (upper panel) as well as those supported by the $\left(\mathrm{N}_{2}\right) / \mathrm{CNT}(5,5)$ and $\left({ }^{4} \mathrm{He}\right) / \mathrm{CNT}(9,9)$ interaction potentials (bottom panel). Left (right) panels show the bound states for the adsorbates inside (outside) the carbon nanotubes. The densities $D(r)$ are normalized as $\int \mathrm{d} r D(r)=1$. All bound states correspond to $m=0$. The experimental values of bound-state energies for ${ }^{4} \mathrm{He} /$ graphite are also depicted with dashed gray lines. ${ }^{56}$

Table 3. Nuclear Bound-State Energies $\left(\epsilon_{m}^{n}\right.$, in $\left.\mathrm{meV}\right)$ of ${ }^{4} \mathrm{He} / \mathrm{CNT}$ and $\mathrm{N}_{2} / \mathrm{CNT}$ Complexes As a Function of Vibrational ( $n$ ) and Rotational $(m)$ Quantum Numbers, with the Adsorbate inside the Tubes ${ }^{a}$

\begin{tabular}{|c|c|c|c|c|c|c|}
\hline$n$ & $m=0$ & $m=1$ & $m=2$ & $m=3$ & $m=4$ & $m=5$ \\
\hline \multicolumn{7}{|c|}{${ }^{4} \mathrm{He} / \mathrm{CNT}(5,5)$ inside } \\
\hline 0 & -66.87 & -55.53 & -43.26 & -30.15 & -16.28 & -1.71 \\
\hline 1 & -42.20 & -28.21 & -13.56 & & & \\
\hline 2 & -12.66 & & & & & \\
\hline \multicolumn{7}{|c|}{${ }^{4} \mathrm{He} / \mathrm{CNT}(9,9)$ inside } \\
\hline 0 & -32.66 & -32.60 & -32.42 & -32.13 & -31.71 & -31.18 \\
\hline 1 & -23.60 & -23.52 & $-23 . .30$ & -22.92 & -22.40 & -21.74 \\
\hline 2 & -16.77 & -16.66 & -16.33 & -15.79 & -15.07 & -14.17 \\
\hline \multicolumn{7}{|c|}{$\mathrm{N}_{2} / \mathrm{CNT}(5,5)$ inside } \\
\hline 0 & -356.99 & -344.19 & -331.86 & -318.22 & -305.05 & -291.76 \\
\hline 1 & -331.14 & -317.97 & -304.68 & -291.27 & -277.75 & -264.11 \\
\hline 2 & -304.55 & -291.02 & -277.38 & -263.63 & -249.77 & -235.81 \\
\hline
\end{tabular}

${ }^{a_{T}}$ The $\epsilon_{m}^{n}$ values are provided for all the ${ }^{4} \mathrm{He} / \mathrm{CNT}(5,5)$ bound states, along with those with $m<6$ and $n<3$ for ${ }^{4} \mathrm{He} / \mathrm{CNT}(9,9)$ and $\mathrm{N}_{2} / \mathrm{CNT}(9,9)$ complexes. Notice that these energies are calculated using long nanotubes (see Figure 3 ) and not the short nanotube considered in the SAPT(DFT) calculations (see Figure 2). 

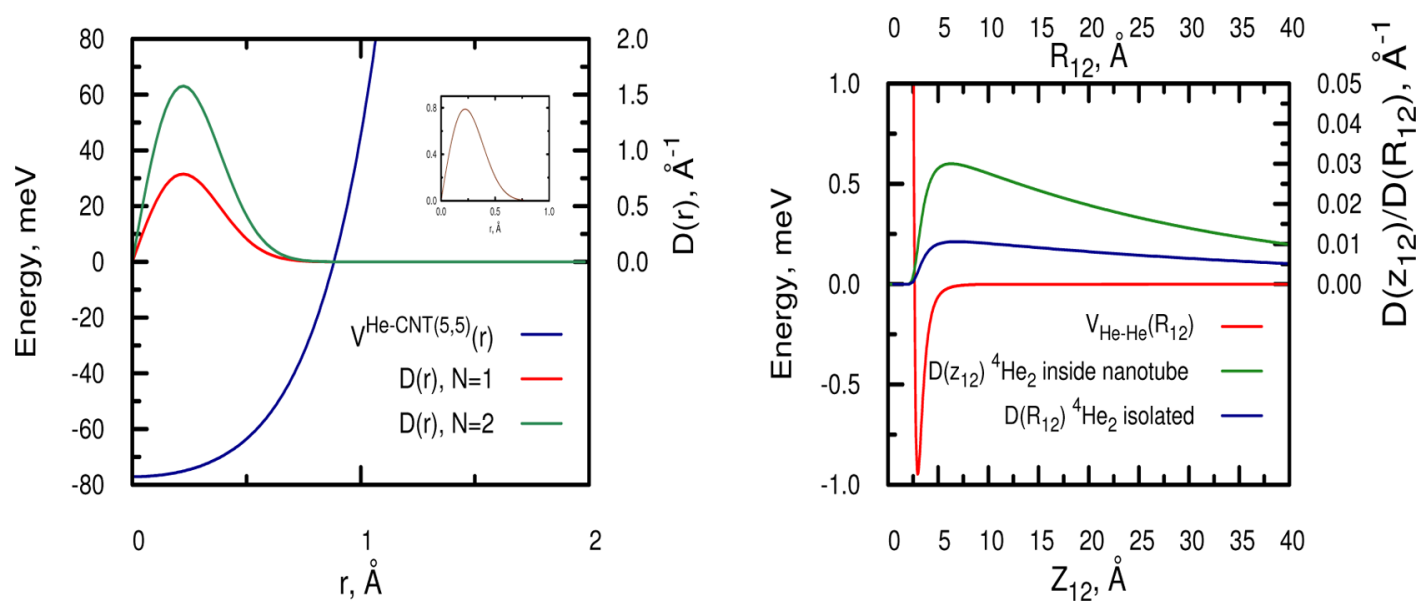

Figure 5. Left panel: $\mathrm{He} / \mathrm{CNT}(5,5)$ interaction potential and radial distributions of densities for $N^{4} \mathrm{He}$ atoms inside the nanotube. The densities are normalized as $\int \mathrm{d} r D(r)=N$ (those shown in the inset are renormalized to unity). Right panel: Comparison of the pair density distribution of the pure ${ }^{4} \mathrm{He}_{2}$ dimer with that of the $(\mathrm{He})_{2} / \mathrm{CNT}(5,5)$ complex as a function of the relative $z_{12}=z_{2}-z_{1}$ distance (scaled to 2.0 to have the same normalization). The $z_{12}$ density is symmetric so that only the region with $z_{12}>0$ is displayed.
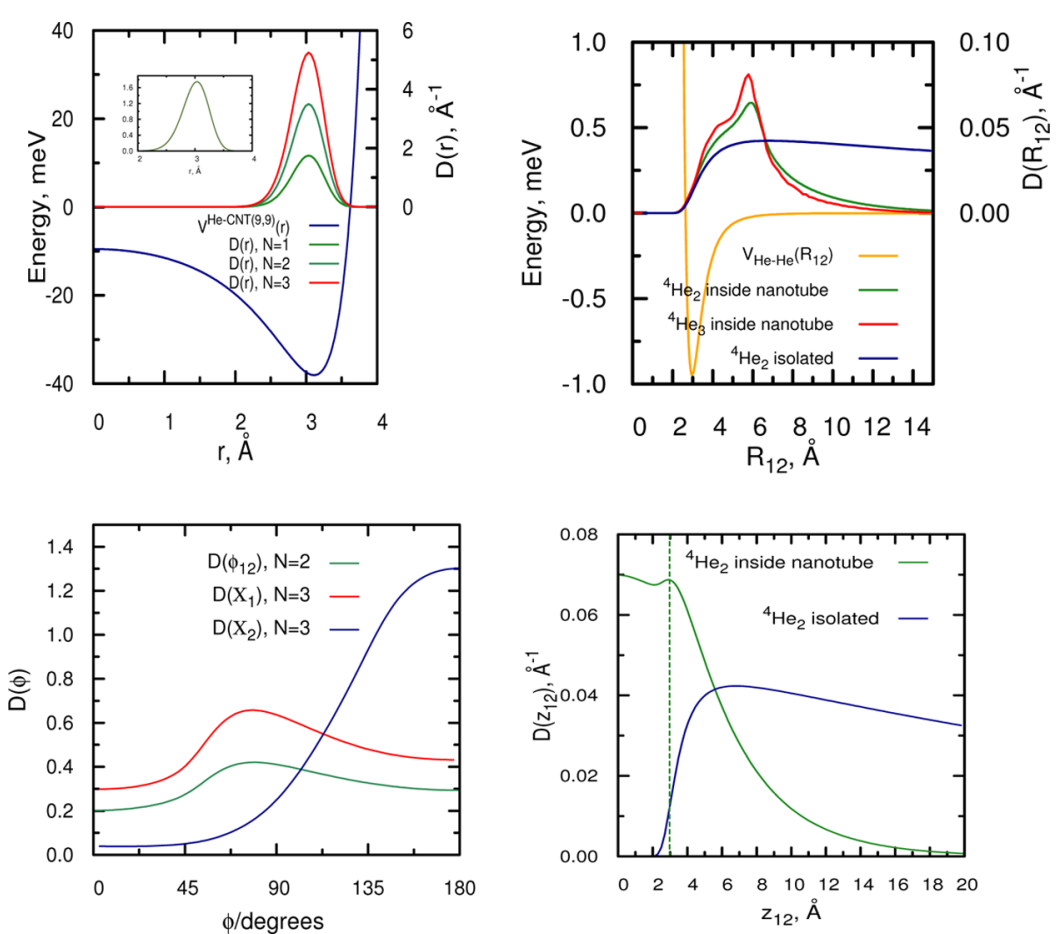

Figure 6. Upper left panel: $\mathrm{He} / \mathrm{CNT}(9,9)$ interaction potential and radial distributions of densities for ${ }^{4} \mathrm{He}$ atoms inside the nanotube. The densities are normalized as $\int \mathrm{d} r D(r)=N$ (those shown in the inset are renormalized to unity). Upper right panel: He-He interaction potential and pair density distributions as a function of the $\mathrm{He}-\mathrm{He}$ distance for $\mathrm{N}^{4} \mathrm{He}$ atoms inside the nanotube and the ${ }^{4} \mathrm{He}_{2}$ dimer. Lower left panel: Pair density distributions as a function of the relative $\phi_{12}=\phi_{2}-\phi_{1}$ angle for $N=2$, and the $\chi_{1}=\phi_{2}-\phi_{1}$ and $\chi_{2}=\phi_{3}-\frac{\phi_{1}+\phi_{2}}{2}$ angles for $N=3$. Lower right panel: Pair density distributions as a function of the relative $z_{12}=z_{2}-z_{1}$ distance. Notice the density peak at about the minimum of the HeHe interaction potential $(\sim 3 \AA)$.

Fixing $\Lambda$ to zero, a single bound state is found when the $\mathrm{He}-$ $\mathrm{He}$ interaction is included. Starting with the $\mathrm{CNT}(5,5)$ tube, the left panel of Figure 5 shows the $N$-dependence of groundstate single-particle radial distributions while pair density distributions as a function of the relative $\mathrm{He}-\mathrm{He}$ distance along $z$ are presented in the right panel. From the inset of Figure 5, notice that the radial distributions renormalized to unity are almost indistinguishable for $N=1-2$, with the peak distributions at the center of the nanotube where the potential minimum is located $(r=0)$. The total wave function can be written as a single product of single-particle wave functions, with $N{ }^{4} \mathrm{He}$ atoms along the $z$ axis. From the right panel of Figure 5, we can see that the pair density distribution is extremely delocalized along the tube long axis so that the average value $\left\langle\left|z_{12}\right|\right\rangle$ is $22.3 \AA$.

As found in previous studies of doped and para- $\mathrm{H}_{2}$ and helium clusters (see, e.g., refs 57-60 and references therein), the total energy of ${ }^{4} \mathrm{He} / \mathrm{CNT}$ complexes can be expressed as $N$ times the energy of the ground-state wave function for $N=1$ (i.e., the lowest nuclear orbital) plus a very small attractive term 


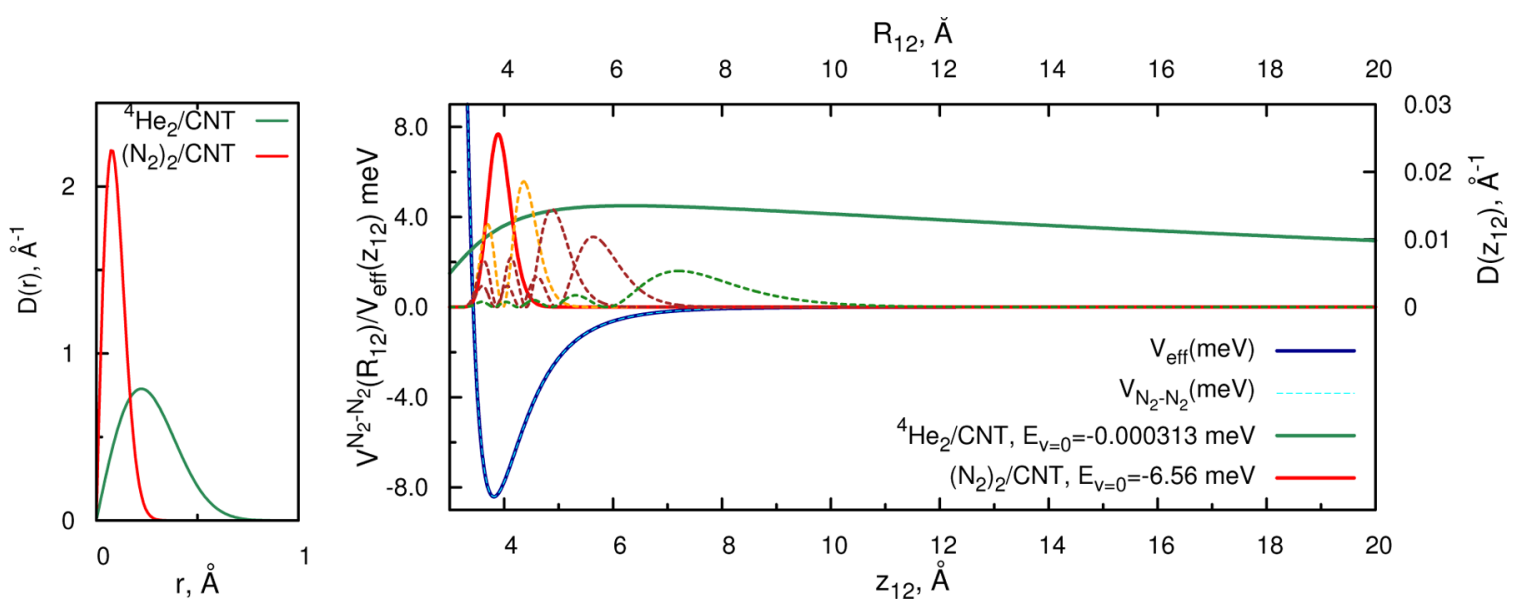

Figure 7. Left panel: radial distribution for two $\mathrm{N}_{2}$ molecules (red) or two ${ }^{4} \mathrm{He}$ atoms (sea-green) in the CNT(5,5) tube. Right panel: pair density distribution $D\left(z_{12}\right)$ as a function of the relative distance along $z$ between two $\mathrm{N}_{2}$ molecules (red) or two ${ }^{4} \mathrm{He}$ atoms (sea-green) in the CNT(5,5) tube. Pair densities for two $\mathrm{N}_{2}$ molecules in excited states (dashed lines) are also shown, along with the $\mathrm{N}_{2}-\mathrm{N}_{2}$ interaction potential of both pure and confined $\left(\mathrm{N}_{2}\right)_{2}$ dimers.

$\left\langle V_{\mathrm{He}-\mathrm{He}}\right\rangle$ arising from the $\mathrm{He}-\mathrm{He}$ interaction, $E_{N}=N \times \epsilon_{0}^{0}+$ $\left\langle V_{\mathrm{He}-\mathrm{He}}\right\rangle$. The binding energy between two ${ }^{4} \mathrm{He}$ atoms inside the $\mathrm{CNT}(5,5)$ tube is only $-0.313 \mu \mathrm{eV}$. This value can be compared with the nuclear bound state energy of the pure ${ }^{4} \mathrm{He}_{2}$ dimer $(-0.138 \mu \mathrm{eV})$, despite the well-depth of the $\mathrm{He}-\mathrm{He}$ potential $(-0.94 \mathrm{meV}$; see Figure 5). Due to the hard core of the $\mathrm{He}-\mathrm{He}$ interaction and very small ${ }^{4} \mathrm{He}$ mass, the ${ }^{4} \mathrm{He}-{ }^{4} \mathrm{He}$ vibrational motion is characterized by an exceptionally large zero point energy as compared with the corresponding welldepth. Thus, the average interatomic separation of the helium dimer is $50 \AA$, which is much larger than the $\mathrm{He}-\mathrm{He}$ distance at the vdW minimum of $3 \AA$. The $\mathrm{He}-\mathrm{He}$ interaction is very weakly screened by the $\operatorname{CNT}(5,5)$ carbon cage so that, in practice, we have an almost unperturbed "pure" ${ }^{4} \mathrm{He}$ dimer along $z$, holding a long-range tail of attractive interaction essentially stopping at $100 \AA$ (see right panel of Figure 5).

From our results, it is clear that the large average interatomic distance of the ${ }^{4} \mathrm{He}_{2}$ dimer (about $50 \AA$ ) along with the weak screening of the $\mathrm{He}-\mathrm{He}$ interaction when the ${ }^{4} \mathrm{He}$ atoms are accommodated along the tube long axis are fundamental reasons why small filling factors have been measured for helium adsorption in small diameter (below $0.7 \mathrm{~nm}$ ) nanotubes. ${ }^{9}$ Due to the larger mass of the $\mathrm{N}_{2}$ molecule and the more attractive pair interaction, the average separation of $\mathrm{N}_{2}$ centers of mass for the $\left(\mathrm{N}_{2}\right)$ dimer (about $4 \AA$, see below) is much shorter than for the ${ }^{4} \mathrm{He}_{2}$ dimer. Hence, it can be expected that the number of $\mathrm{He}$ atoms in a given length is smaller than the number of $\mathrm{N}_{2}$ molecules. This would already explain why $\mathrm{N}_{2}$ adsorption is less restricted in narrow nanotubes; ${ }^{9}$ vide infra for NWF calculations supporting this assumption. Since helium adsorption was found unimpeded through larger diameter (above 0.7 $\mathrm{nm}$ ) nanopores, it is expected that the effective ${ }^{4} \mathrm{He}-{ }^{4} \mathrm{He}$ motion must be strongly dependent on the confinement size, i.e. the nanotube radius. To prove this, let us consider the $\operatorname{CNT}(9,9)$ tube with a diameter of $\sim 1.1 \mathrm{~nm}$ (see Figure 6). As for the $\operatorname{CNT}(5,5)$, it turns out that $N{ }^{4} \mathrm{He}$ atoms occupy the same orbital (i.e., the ground-state wave function of a single ${ }^{4} \mathrm{He}$ atom). Consequently, the radial density distributions, $D(r)$, differ just by the normalization factor $N$ (see upper left panel of Figure 6). The ${ }^{4} \mathrm{He}$ atoms are confined at the potential minimum, which, for the larger tubes, is located at the lateral region closer to the carbon wall (see Figure 3).
Once again, the total energy for $N{ }^{4} \mathrm{He}$ atoms inside the $\operatorname{CNT}(9,9)$ tube can be written as $N$ times the energy of the occupied orbital, $\epsilon_{0}^{0}$, plus a term $\left\langle V_{\mathrm{He}-\mathrm{He}}\right\rangle$ accounting for the weakly attractive $\mathrm{He}-\mathrm{He}$ interaction. The binding energy is -0.0224 and $-0.0353 \mathrm{meV}$ for two and three ${ }^{4} \mathrm{He}$ atoms, respectively, i.e., 2 orders of magnitude larger than for either the $\mathrm{CNT}(5,5)$ counterpart $(-0.313 \mu \mathrm{eV})$ or the isolated ${ }^{4} \mathrm{He}$ dimer $(-0.138 \mu \mathrm{eV})$. Comparing the pair density distributions of an isolated and confined ${ }^{4} \mathrm{He}$ dimer (see upper right panel of Figure 6), it is clear that the latter is much more localized. The pair density is peaked at $r_{\mathrm{He}-\mathrm{He}} \sim 6 \AA$, which is close to $2 r_{e}(6.4$ $\AA$ ). Besides the maximum, notice the shoulder of the pair density entering into the minimum region of the $\mathrm{He}-\mathrm{He}$ potential. As shown in the Supporting Information, the pair correlation function is peaked at the same $\mathrm{He}-\mathrm{He}$ distance as measured in bulk superfluid liquid helium by neutron diffraction. ${ }^{61}$ Furthermore, when going from two to three ${ }^{4} \mathrm{He}$ atoms, the pair function develops a minimum at the same position as the liquid helium counterpart.

The pair distribution over the relative $\phi_{12}$ angle (see lower left panel of Figure 6) displays the maximum for the ${ }^{4} \mathrm{He}_{2}$ dimer in an orthogonal orientation to the nanotube axis. A third ${ }^{4} \mathrm{He}$ atom finds its optimal position at the opposite site of the ${ }^{4} \mathrm{He}_{2}$ dimer center of mass. In turn (see the lower right panel of Figure 6), the pair distribution over the relative $z_{12}$ distance is peaked at zero, i.e., with the ${ }^{4} \mathrm{He}$ atoms in the plane perpendicular to the tube axis. However, it is important to notice the bending of the distribution at the position of the minimum for the $\mathrm{He}-\mathrm{He}$ potential $(\sim 2.97 \AA)$ and the long tail extending up to $20 \AA$. These features indicate the twodimensional confinement exerted by the carbon cage. Although the obvious most probable configuration for three ${ }^{4} \mathrm{He}$ atoms in the perpendicular plane would be triangular, pair density distributions reflect very wide amplitude motions. This is particularly evident by the delocalization of the ${ }^{4} \mathrm{He}-{ }^{4} \mathrm{He}$ nuclear wave function in $z$. The coupling between the motions in $z_{12}$ and $\phi_{12}$ is also noteworthy.

Summarizing, the ${ }^{4} \mathrm{He}$ atoms fill a toroidal two-dimensional shell around and along the $\operatorname{CNT}(9,9)$ tube symmetry axis. As opposed to the $\operatorname{CNT}(5,5)$ case, there is no bound state with the ${ }^{4} \mathrm{He}$ atoms at the tube center, despite the attractive $\mathrm{He} /$ $\mathrm{CNT}(9,9)$ interaction there; see Supporting Information for an 

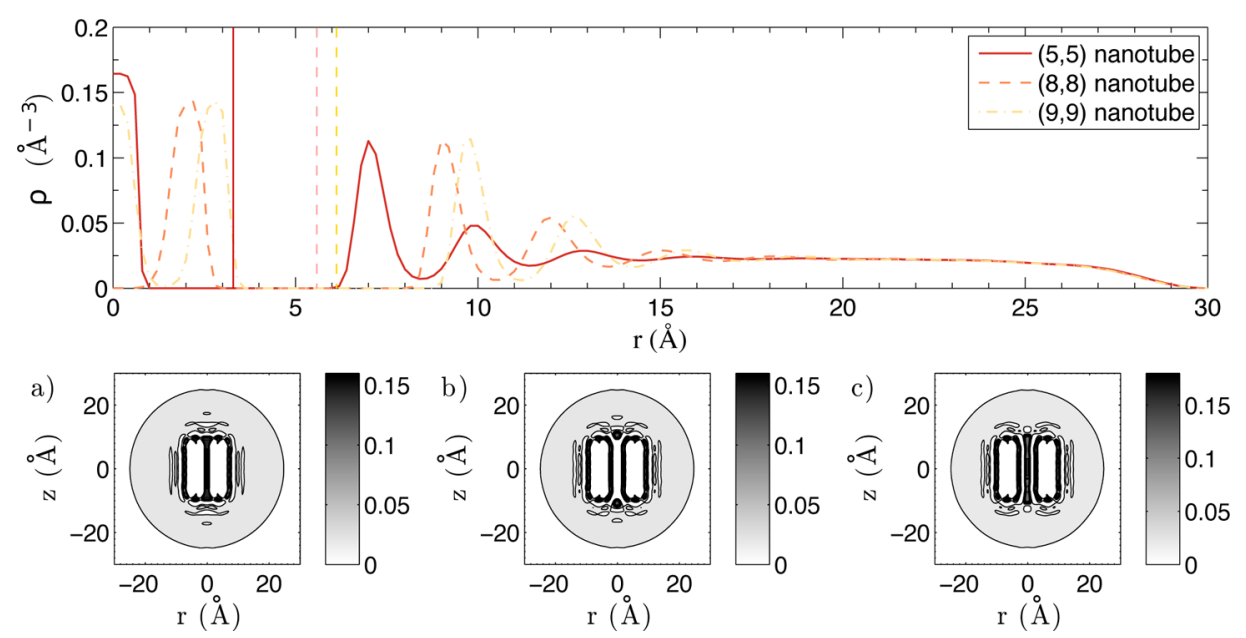

Figure 8. Upper panel: radial distribution of the helium densities for fully immersed carbon nanotubes at $z=0$. Positions of the tube walls are indicated by vertical bars of the same color. Lower panel: Contour plots of the helium density for fully submerged carbon nanotubes of various helicities $(n, n)$, for $n=5,8$, and 9, labeled in ascending order from (a) to (c).

effective interaction model along $z$ explaining this behavior. The average radius of the toroidal helium shell can be identified with the $\mathrm{r}_{e}$ value at the potential minimum (i.e., $r_{e}=3.2 \AA$; see Table 2). Taking into account the position of the highest peak in the $D\left(R_{\mathrm{He}-\mathrm{He}}\right)$ distribution (about $6.0 \AA \AA$; see Figure 6), it is clear that the shell is not saturated with three ${ }^{4} \mathrm{He}$ atoms. Since the wave function calculation becomes unfeasible for larger sizes, we will get back to the density functional formulation of Paper $\mathrm{I}^{21}$ (see next section).

4.3. Comparison between ${ }^{4} \mathrm{He}_{2} / \mathrm{CNT}(5,5)$ and $\left(\mathrm{N}_{2}\right)_{2} /$ CNT $(5,5)$ Complexes. The main reason why a nanotube of diameter below $0.7 \mathrm{~nm}$ is better filled with $\mathrm{N}_{2}$ than with helium at low temperature can be revealed by comparing the nuclear wave functions of ${ }^{4} \mathrm{He}_{2} / \mathrm{CNT}(5,5)$ and $\left(\mathrm{N}_{2}\right)_{2} / \mathrm{CNT}(5,5)$ complexes in the ground state. Here we employ the $\mathrm{N}_{2}-\mathrm{N}_{2}$ interaction potential of ref 11 . Radial density distributions $D(r)$ and pair density distributions $D\left(z_{12}\right)$ are shown in Figure 7 . It can be seen that the nuclear wave function is much more localized for $\left(\mathrm{N}_{2}\right)_{2}$ than for ${ }^{4} \mathrm{He}_{2}$, either in the radial direction $r$ or along the longitudinal $z$ axis of the nanotube.

For both ${ }^{4} \mathrm{He}_{2} / \mathrm{CNT}(5,5)$ and $\left(\mathrm{N}_{2}\right)_{2} / \mathrm{CNT}(5,5)$ systems, the dimer nuclear motion along $r$ is decoupled from the relative motion of the monomers along $z$. Then, the total wave function is simply a product of two monomer single-particle wave functions, located at a certain distance along $z$. Once again, the total energy can be expressed as two times the energy of the single $\mathrm{N}_{2} / \mathrm{CNT}$ complex, $\epsilon_{0}^{0}$, plus a term, $\left\langle V_{\mathrm{N}_{2}-\mathrm{N}_{2}}\right\rangle$, coming from the effective $\mathrm{N}_{2}-\mathrm{N}_{2}$ interaction along $z$. As a zeroth-order approximation (see Supporting Information for the details), this effective interaction can be written as a function of the relative $z_{12}$ distance as

$$
V_{\text {eff }}\left(z_{12}\right)=\frac{1}{2 \pi} \int_{0}^{2 \pi} \mathrm{d} \phi_{12} V_{\mathrm{N}_{2}-\mathrm{N}_{2}}(R)
$$

where $V_{\mathrm{N}_{2}-\mathrm{N}_{2}}$ is the interaction potential of the pure $\mathrm{N}_{2}-\mathrm{N}_{2}$ dimer and $R$ is defined as

$$
R^{2}=2 r_{0}^{2}\left(1-\cos \phi_{12}\right)+z_{12}^{2}
$$

with $r_{0}$ as the $r$ average over the radial distribution $D(r)$ (about $0.096 \AA$ ). The strong confinement in short diameter nanotubes leads to very small $r_{0}$ values.
From eq 10, notice that the interaction potential of two isolated $\mathrm{N}_{2}$ molecules $V_{\mathrm{N}_{2}-\mathrm{N}_{2}}$ (or two isolated $\mathrm{He}$ atoms) is slightly modified by the carbon cage. The effective $V_{\text {eff }}$ and unperturbed interaction potential $V_{\mathrm{N}_{2}-\mathrm{N}_{2}}$, plotted together in Figure 7 , are almost indistinguishable. Hence, the term $\left\langle V_{\mathrm{N}_{2}-\mathrm{N}_{2}}\right\rangle$ can be estimated as the energy of the lowest vibrational level supported by the unperturbed $V_{\mathrm{N}_{2}-\mathrm{N}_{2}}$ potential. The same holds true for excited states sharing the same $D(r)$ distribution (i.e., the five bound states of the $V_{\mathrm{N}_{2}-\mathrm{N}_{2}}$ potential).

Using our effective model (see eq 10), "exact" and estimated $\left\langle V_{\mathrm{N}_{2}-\mathrm{N}_{2}}\right\rangle$ values are -6.55 and $-6.56 \mathrm{meV}$, respectively, while "exact" and estimated $\left\langle V_{\mathrm{He}-\mathrm{He}}\right\rangle$ values are -0.313 and -0.284 $\mu \mathrm{eV}$. The $\mathrm{N}_{2}-\mathrm{N}_{2}$ binding energy $(-6.55 \mathrm{meV})$ is thus more than 3 orders of magnitude larger than the ${ }^{4} \mathrm{He}-{ }^{4} \mathrm{He}$ counterpart (i.e., $-0.313 \mu \mathrm{eV}$ ). On one hand, the $\mathrm{N}_{2}-\mathrm{N}_{2}$ well is about ten times deeper $(-8.41 \mathrm{vs}-0.94 \mathrm{meV})$. On the other hand, the reduced mass of the $\left(\mathrm{N}_{2}\right)_{2}$ "diatomic" is a factor of 7 larger. Due mainly to the very different scales of quantum zero point effects, the ${ }^{4} \mathrm{He}_{2} / \mathrm{CNT}(5,5)$ system is extremely weakly bound and characterized by an extremely broad distribution in $z$, extending up to $100 \AA$. Contrarily, the $\left(\mathrm{N}_{2}\right)_{2} / \mathrm{CNT}(5,5)$ is still a vdW-type complex, but much more strongly bound than the ${ }^{4} \mathrm{He}_{2} / \mathrm{CNT}(5,5)$ system, with a very localized profile in $z$ and a tail stopping at $5 \AA$. While we find a single bound state for the ${ }^{4} \mathrm{He}_{2} / \mathrm{CNT}(5,5)$ system, a significant number of them exists in the $\left(\mathrm{N}_{2}\right)_{2} / \mathrm{CNT}(5,5)$ case (see Figure 7).

To sum up, our comparison clearly demonstrates that the effective size of the ${ }^{4} \mathrm{He}_{2}$ dimer in narrow nanotubes is much larger than the $\left(\mathrm{N}_{2}\right)_{2}$ counterpart, both of them being confined along the tube long axis and almost unperturbed by the carbon cage. Comparing the average separation of the former and the latter in the ground state (about 50 vs $4 \AA$ ), it follows that the number of $\mathrm{He}$ atoms in a given nanotube length should be much smaller. From this, it can be easily understood why more molecular nitrogen than helium is adsorbed in narrow carbon nanopores at low temperature. ${ }^{9}$

Before closing this section, we briefly comment on the main differences of the $\left(\mathrm{N}_{2}\right)_{2}$ bound states when the nanotube diameter is increased. To analyze them, the Supporting 

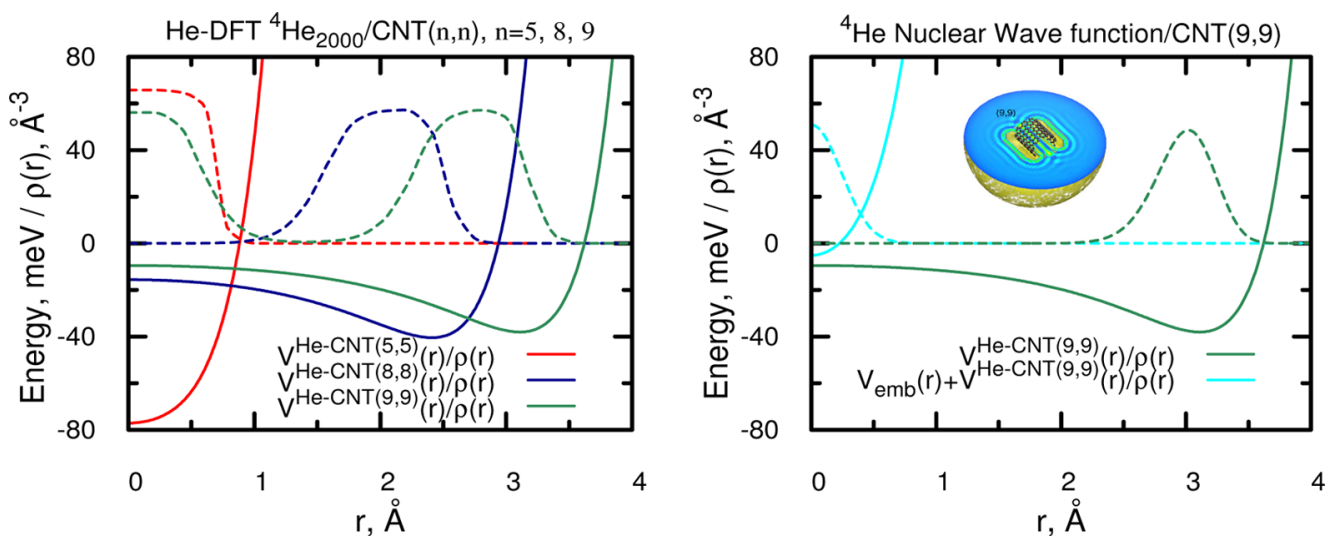

Figure 9. Left panel: Radial distribution of one ${ }^{4} \mathrm{He}_{2000}$ droplet for fully immersed carbon nanotubes of several chiral indices at $z=0$. Right panel: $\mathrm{He} / \mathrm{CNT}(9,9)$ interaction potential at $z=0$ along with radial density of the ${ }^{4} \mathrm{He}$ ground-state wave function (shown in sea-green). Notice that, adding the embedding potential from the second and following helium layers (see inset picture), the global potential minimum (shown in cyan at $z=$ $0)$ is shifted to the center of the nanotube, as the ${ }^{4} \mathrm{He}$ wave function is.

Information reports the details of the lowest-energy bound states of the $\left(\mathrm{N}_{2}\right)_{2} / \mathrm{CNT}(9,9)$ complex. As opposed to the $\mathrm{CNT}(5,5)$ case, a strong coupling between $\phi_{12}$ and $z_{12}$ variables occurs in the wave function for all bound states considered. There is a clear transition from one- to two-dimensional confinement regime, where the density of bound states is much higher. For instance, the energy difference between the ground and the fourth excited state is $-0.72 \mathrm{meV}$ only. Even at $T=5$ $\mathrm{K}$, assuming a Boltzmann distribution, four lowest-energy states would be significantly populated. Contrarily, for the $\left(\mathrm{N}_{2}\right)_{2}$ / $\mathrm{CNT}(5,5)$ complex, the same holds true at $T=50 \mathrm{~K}$. Similarly to the $\operatorname{CNT}(5,5)$ case, all density distributions show rather localized profiles as compared with the helium distributions.

\section{MIXED HELIUM DENSITY FUNCTIONAL AND NUCLEAR WAVE-FUNCTION APPROACH}

Using our new potential model, the He-DFT approach has been applied to a ${ }^{4} \mathrm{He}_{2000}$ droplet with immersed carbon nanotubes of the same chiral indexes as in the wave function calculations; see Supporting Information for additional nanotube sizes. Figure 8 shows one- and two-dimensional distributions of the helium densities along the $r$ and $z$ coordinates. These profiles are very similar to those presented in Paper $\mathrm{I}^{21}{ }^{21}$ with the exception of a shift $(\sim 0.4 \AA)$ for the helium shells outside the nanotube; see Supporting Information for a graphical comparison. In the outer region, the hard-core of the atom-bond model model potential used in ref 21 was located at shorter distances than the $a b$ initio interaction energies. In any case, the similarity of the density profiles illustrates the robustness of the He-DFT results against improvements of the potential model.

Let us now discuss the He-DFT results in the context of the nuclear wave function calculations presented in the previous section. First, we notice that the assumption of condensation in the same $N$-occupied orbital in He-DFT formulations is supported by the calculated nuclear wave functions for $N \leq$ 3. In fact, we have previously shown that renormalized radial densities $D(r)$ are indistinguishable from each other, irrespective of the $N$ value and the nanotube size. This spatial confinement in the radial direction is the key to explain the formation of cavities inside the tube. ${ }^{21}$ Second, as seen in Figure 8 , the $\operatorname{CNT}(5,5)$ tube is saturated with a quasi-onedimensional helium layer along the $z$ axis at $r=0$, i.e, the position of the global $\mathrm{He} / \mathrm{CNT}(5,5)$ potential minimum. Integrating the helium density over the nanotube volume, it is found that just three ${ }^{3} \mathrm{He}$ atoms are filling the $\mathrm{CNT}(5,5)$ tube. Such a small helium filling factor is easily understood by considering the tube length $(\sim 13.5 \AA)$ and extremely extended pair ${ }^{4} \mathrm{He}-{ }^{4} \mathrm{He}$ density distribution along $z$ (beyond $40 \AA$, see right panel of Figure 5). As discussed above, one-dimensional confinement strained by the carbon $(5,5)$ cage together with the huge zero-point energy of the ${ }^{4} \mathrm{He}-{ }^{4} \mathrm{He}$ vibrational motion are mainly responsible for this behavior.

Upon increasing the nanotube radius beyond $0.7 \mathrm{~nm}$ (see Figure 8), an inner concentric helium shell around the tube axis is formed in which about $10-12{ }^{4} \mathrm{He}$ atoms are equally distributed over the $\phi$ direction, with $2-3{ }^{4} \mathrm{He}$ atoms being extended along the nanotube length $(\sim 13.5 \AA)$. As discussed above (see Figure 6), the pair ${ }^{4} \mathrm{He}-{ }^{4} \mathrm{He}$ density distributions are rather localized in the radial direction as compared with the very pronounced nuclear delocalization along $z$. As for helium densities on the outside of the nanotube (see Figure 8 ), the formation of three toroidal helium layers is in good agreement with the filling of the three lowest-energy orbitals supported by the $\mathrm{He} /$ nanotube potential (see Figure 4), considering the effective radii of the cylindric layers and optimal $\mathrm{He}-\mathrm{He}$ intralayer distance.

Overall, nuclear wave function-based and He-DFT pictures translate very well into each other. However, an important feature cannot be explained through our wave function calculations: As seen in Figure 8 and stressed in Paper I, ${ }^{21}$ along the concentric helium shell inside the $\operatorname{CNT}(9,9)$ tube, a central layer does appear with about $2-3{ }^{4} \mathrm{He}$ atoms inside. This is in contrast with both the $\operatorname{CNT}(8,8)$ case (see Figure 8) and the wave function calculations for the $\operatorname{CNT}(9,9)$ tube. To explain this, we apply an embedding scheme, described in the second section, and calculate the wave function of a single ${ }^{4} \mathrm{He}$ atom in an effective potential incorporating the effect of the toroidal helium shells. As shown in Figure 9, the addition of these shells as an embedding potential causes the shift of the global potential minimum from the lateral region to the nanotube center and, then, of the ${ }^{4} \mathrm{He}$ atom wave function. The binding energy of this ${ }^{4} \mathrm{He}$ atom is just $-0.604 \mathrm{meV}$. This indicates that the attractive $\mathrm{He}-\mathrm{CNT}$ interaction at the tube center is effectively screened by the helium shell close to the carbon wall. 


\section{CONCLUSIONS}

The main motivation of this work has been to provide fundamental insights into the role of intermolecular interactions and quantum nuclear effects on molecular adsorption in carbon nanotubes. Due to the availability of experimental measurements, ${ }^{9}$ and as a follow-up study of a theoretical helium density functional-based work, ${ }^{21}$ we have chosen clusters of ${ }^{4} \mathrm{He}$ and $\mathrm{N}_{2}$. Our methodological protocol combines modern $a b$ initio theory for intermolecular interactions, applying the SAPT(DFT) treatment, and our ad-hoc-developed nuclear wave function-based method.

As expected from the larger number of electrons and the polarizability of $\mathrm{N}_{2}$, the SAPT(DFT)-based analysis reveals that the $\mathrm{N}_{2}$ molecule is more strongly confined by the carbon cage than atomic helium, with the $\mathrm{N}_{2} / \mathrm{CNT}$ well being a factor of 46 more attractive. With both $\mathrm{He} / \mathrm{CNT}$ and $\mathrm{N}_{2} / \mathrm{CNT}$ being dispersion-dominated interactions, the attractive electrostatic component from the quadruple-quadruple interaction is significant for the $\mathrm{N}_{2}$ /nanotube and absent for the $\mathrm{He}$ / nanotube pair. Upon increasing the nanotube diameter, the potential minima move from the center to positions closer to the carbon wall.

The main question raised by experiment ${ }^{9}$ was why helium barely adsorbs in small diameter carbon nanopores (below 0.7 $\mathrm{nm})$ at low temperature $(T=2-5 \mathrm{~K})$ while $\mathrm{N}_{2}$ diffuses more easily, a finding which is in stark contrast with the classical expectation based on kinetic diameters. As already indicated in the original experimental study ${ }^{9}$ and confirmed by our helium density functional studies, ${ }^{21}$ our benchmark calculations show that this can be attributed to a larger effective size of helium compared to $\mathrm{N}_{2}$ caused by more marked nuclear quantum effects at the low-temperature regime. Selecting a nanotube with diameter below $0.7 \mathrm{~nm}$ with two $\mathrm{N}_{2}$ molecules or ${ }^{4} \mathrm{He}$ atoms inside, we have demonstrated the carbon-cage-induced confinement is one-dimensional along the tube longitudinal $z$ axis and that the effective $\mathrm{He}-\mathrm{He}$ and $\mathrm{N}_{2}-\mathrm{N}_{2}$ interactions are almost unperturbed from that of the isolated dimers. We propose an effective one-dimensional model explaining this finding. The very different average ${ }^{4} \mathrm{He}-{ }^{4} \mathrm{He}$ and $\mathrm{N}_{2}-\mathrm{N}_{2}$ separations (about 50 and $4 \AA$, respectively) explain why the number of adsorbed ${ }^{4} \mathrm{He}$ atoms at a given nanotube length should be smaller than the number of $\mathrm{N}_{2}$ molecules, despite the expected variation in adsorbate clusters larger than dimers.

Both the interaction adsorbate/nanotube potential and the nuclear wave function landscapes change completely when the adsorbates are confined in nanotubes of larger diameter (above $0.7 \mathrm{~nm}$ ). First, the global minimum of the potential shifts from the nanotube center to the lateral region closer to the carbon cage. This causes the turnover to a two-dimensional confinement regime for which the ${ }^{4} \mathrm{He}$ atoms start to fill a toroidal helium shell around the tube long axis. Moreover, the pair correlation functions start to have profiles resembling that measured for bulk superfluid liquid helium at $T=1 \mathrm{~K}^{61}$ The radial densities from nuclear wave function and helium density functional calculations are fully consistent with each other. ${ }^{21}$ Importantly, the nuclear wave function calculations confirm that the ${ }^{4} \mathrm{He}$ atoms occupy the same single-particle orbital in the radial direction of different sized nanotubes, providing the key explanation for the existence of cavities of zero helium density. ${ }^{21}$ The application of our embedding approach which combines both methods is necessary, however, to explain the appearance of an additional helium accumulation along the tube long axis. When considering $\mathrm{N}_{2}$ as the adsorbate, the density of bound states is much higher in the two-dimensional confinement regime. For both helium and $\mathrm{N}_{2}$, the quantum modes associated with angular degrees of freedom become strongly coupled to the motion along $z$ under two-dimensional confinement.

Being aware of the importance of accurate modeling for the adsorption of gas molecules onto carbon nanostructures, we have proposed a pairwise additive model which also bears the potential for generalization to other types of gas molecules. Our work also illustrates how nuclear density functional and wave function treatments can be complementary to each other. Work is in progress to further advance in the embedding approach proposed in this work, opening the possibility to reveal deeper insights into the coupled motions of large molecular quantum systems via a combination of helium density functional- and nuclear wave function-based treatments.

\section{ASSOCIATED CONTENT}

\section{Supporting Information}

The Supporting Information is available free of charge on the ACS Publications website at DOI: 10.1021/acs.jpcc.6b12959.

Details of the nuclear wave function-based approach, $\mathrm{He}-\mathrm{He}$ pair correlation functions, a model to estimate the effective adsorbate-adsorbate interaction along the tube long axis, density distributions and energies of the $\left(\mathrm{N}_{2}\right)_{2} / \mathrm{CNT}(9,9)$ complex, helium densities from the application of the density functional approach to carbon nanotubes with helicity indexes from $(4,4)$ to $(9,9)$, and two tables with model parameters for the $\mathrm{He} / \mathrm{CNT}$ and $\mathrm{N}_{2} / \mathrm{CNT}$ interactions (PDF)

\section{AUTHOR INFORMATION}

\section{Corresponding Author}

*E-mail: Pilar.deLara.Castells@csic.es.

ORCID ${ }^{\circ}$

Andreas W. Hauser: 0000-0001-6918-3106

María Pilar de Lara-Castells: 0000-0001-8697-5770

Notes

The authors declare no competing financial interest.

\section{ACKNOWLEDGMENTS}

This work has been partly supported by the COST Action CM1405 "Molecules in Motion" (MOLIM) and MICINN (Spain) under Grant No. MAT2016-753354-P. MPdLC thanks the CTI (CSIC) and CESGA supercomputer facilities (Spain) for the resources provided.

\section{REFERENCES}

(1) Laird, E. A.; Kuemmeth, D.; Steele, G. A.; Grove-Ramussen, K.; Nygărd, J.; Flensberg, K.; Kouwenhoven, L. P. Quantum Transport in Carbon Nanotubes. Rev. Mod. Phys. 2015, 87, 703-764.

(2) Baughman, R. H.; Zakhidov, A. A.; de Heer, W. A. Carbon Nanotubes-the Route Toward Applications. Science 2002, 297, 787792.

(3) Kowalczyk, P.; Terzyk, A. P.; Gauden, P. A.; Furmaniak, S.; Kaneko, K.; Miller, T. F., III Nuclear Quantum Effects in the Layering and Diffusion of Hydrogen Isotopes in Carbon Nanotubes. J. Phys. Chem. Lett. 2015, 6, 3367-3372.

(4) Nguyen, T. T.; Serp, P. Confinement of Metal Nanoparticles in Carbon Nanotubes. ChemCatChem 2013, 5, 3595-3603. 
(5) Hauser, A. W.; Schwerdtfeger, P. Nanoporous Graphene Membranes for Efficient ${ }^{3} \mathrm{He} /{ }^{4} \mathrm{He}$ Separation. J. Phys. Chem. Lett. 2012, 3, 209-213.

(6) Hauser, A. W.; Schrier, J.; Schwerdtfeger, P. Helium Tunneling through Nitrogen-Functionalized Graphene Pores: Pressure- and Temperature-Driven Approaches to Isotope Separation. J. Phys. Chem. C 2012, 116, 10819-10827.

(7) Bartolomei, M.; Carmona-Novillo, E.; Hernández, M. I.; Campos-Martínez, J.; Pirani, F.; Giorgi, G. Graphdiyne Pores: Ad Hoc Openings for Helium Separation Applications. J. Phys. Chem. C 2014, 118, 29966-29972.

(8) Hernández, M. I.; Bartolomei, M.; Campos-Martínez, J. Transmission of Helium Isotopes through Graphdiyne Pores: Tunneling versus Zero Point Energy Effects. J. Phys. Chem. A 2015, 119, 10743-10749.

(9) Ohba, T. Limited Quantum Helium Transportation Through Nano-Channels by Quantum Fluctuation. Sci. Rep. 2016, 6, 28992.

(10) Cencek, W.; Przybytek, M.; Komasa, J.; Mehl, J. B.; Jeziorski, B.; Szalewicz, K. Effects of Adiabatic, Relativistic, and Quantum Electrodynamics Interactions on the Pair Potential and Thermophysical Properties of Helium. J. Chem. Phys. 2012, 136, 224303.

(11) Cappelletti, D.; Pirani, F.; Bussery-Honvault, B.; Gomez, L.; Bartolomei, M. A Bond-Bond Description of the Intermolecular Interaction Energy: the Case of Weakly Bound $\mathrm{N}_{2}-\mathrm{H}_{2}$ and $\mathrm{N}_{2}-\mathrm{N}_{2}$ Complexes. Phys. Chem. Chem. Phys. 2008, 10, 4281-4293.

(12) Berg, R. F. Simple Flow Meter and Viscometer of High Accuracy for Gases. Metrologia 2005, 42, 11-23.

(13) Berg, R. F. Simple Flow Meter and Viscometer of High Accuracy for Gases (Erratum). Metrologia 2006, 43, 183.

(14) de Lara-Castells, M. P.; Stoll, H.; Mitrushchenkov, A. O. Assessing the Performance of Dispersionless and DispersionAccounting Methods: Helium Interaction with Cluster Models of the $\mathrm{TiO}_{2}(110)$ Surface. J. Phys. Chem. A 2014, 118, 6367-6384.

(15) de Lara-Castells, M. P.; Stoll, H.; Civalleri, B.; Causà, M.; Voloshina, E.; Mitrushchenkov, A. O.; Pi, M. Communication: A Combined Periodic Density Functional and Incremental WaveFunction-Based Approach for the Dispersion-Accounting TimeResolved Dynamics of ${ }^{4} \mathrm{He}$ Nanodroplets on Surfaces: ${ }^{4} \mathrm{He} /$ Graphene. J. Chem. Phys. 2014, 141, 151102.

(16) de Lara-Castells, M. P.; Mitrushchenkov, A. O.; Stoll, H. Combining Density Functional and Incremental Post-Hartree-Fock Approaches for van der Waals Dominated Adsorbate-Surface Interactions: $\mathrm{Ag}_{2} /$ Graphene. J. Chem. Phys. 2015, 143, 102804.

(17) de Lara-Castells, M. P.; Aguirre, N. F.; Stoll, H.; Mitrushchenkov, A. O.; Mateo, D.; Pi, M. Communication: Unraveling the ${ }^{4} \mathrm{He}$ Droplet-mediated Soft-landing from $\mathrm{Ab}$ initio-Assisted and Time-Resolved Density Functional Simulations: $\mathrm{Au} @{ }^{4} \mathrm{He}_{300} /$ $\mathrm{TiO}_{2}(110)$. J. Chem. Phys. 2015, 142, 131101.

(18) de Lara-Castells, M. P.; Bartolomei, M.; Mitrushchenkov, A. O.; Stoll, H. Transferability and Accuracy by Combining Dispersionless Density Functional and Incremental Post-Hartree-Fock Theories: Noble gases Adsorption on Coronene/Graphene/Graphite Surfaces. J. Chem. Phys. 2015, 143, 194701.

(19) de Lara-Castells, M. P.; Fernández-Perea, R.; Madzharova, F.; Voloshina, E. Post-Hartree-Fock Studies of the $\mathrm{He} / \mathrm{Mg}(0001)$ Interaction: Anti-corrugation, Screening and Pairwise Additivity. J. Chem. Phys. 2016, 144, 244707.

(20) Tamijani, A. A.; Salam, A.; de Lara-Castells, M. P. Adsorption of Noble-Gas Atoms on the $\mathrm{TiO}_{2}(110)$ Surface: An Ab Initio-Assisted Study with van der Waals-Corrected DFT. J. Phys. Chem. C 2016, 120, 18126-18139.

(21) Hauser, A. W.; de Lara-Castells, M. P. Carbon Nanotubes Immmersed in Superfluid Helium: The Impact of Quantum Confinement on Wetting and Capillary Action. J. Phys. Chem. Lett. 2016, 7, 4929-4935.

(22) Hauser, A. W.; de Lara-Castells, M. P. Spatial Quenching of a Molecular Charge-Transfer Process in a Quantum Fluid: The $\mathrm{Cs}_{x}-\mathrm{C}_{60}$ Reaction in Superfluid Helium Nanodroplets. Phys. Chem. Chem. Phys. 2017, 19, 1342-1351.
(23) Smith, D. G. A.; Patkowski, K. Benchmarking the $\mathrm{CO}_{2}$ Adsorption Energy on Carbon Nanotubes. J. Phys. Chem. C 2015, 119, 4934-4948.

(24) Bartolomei, M.; Carmona-Novillo, E.; Hernández, M. I.; Campos-Martínez, J.; Pirani, F. Global Potentials for the Interaction Between Rare Gases and Graphene-Based Surfaces: An Atom-Bond Pairwise Additive Representation. J. Phys. Chem. C 2013, 117, 1051210522.

(25) Misquitta, A. J.; Jeziorski, B.; Szalewicz, K. Dispersion Energy from Density-Functional Theory Description of Monomers. Phys. Rev. Lett. 2003, 91, 033201.

(26) Heßelmann, A.; Jansen, G. Intermolecular Dispersion Energies from Time-Dependent Density Functional Theory. Chem. Phys. Lett. 2003, 367, 778-784.

(27) Dalfovo, F.; Lastri, A.; Pricaupenko, L.; Stringari, S.; Treiner, J. Structural and Dynamical Properties of Superfluid Helium: A DensityFunctional Approach. Phys. Rev. B: Condens. Matter Mater. Phys. 1995, 52, 1193.

(28) Barranco, M.; Guardiola, R.; Hernández, S.; Mayol, R.; Navarro, J.; Pí, M. Helium Nanodroplets: an Overview. J. Low Temp. Phys. 2006, $142,1-81$.

(29) Volkoff, T.; Kwon, Y.; Whaley, K. B. Path Integral Monte Carlo Simulations of Global and Local Superfluidity in Liquid ${ }^{4} \mathrm{He}$ Reservoirs Separated by Nanoscale Apertures. Phys. Rev. B: Condens. Matter Mater. Phys. 2016, 94, 144510.

(30) Del Maestro, A.; Boninsegni, M.; Affleck, I. ${ }^{4} \mathrm{He}$ Luttinger Liquid in Nanopores. Phys. Rev. Lett. 2011, 106, 105303.

(31) Rossi, M.; Ancilotto, F. Superfluid Behavior of Quasi-OneDimensional $p-\mathrm{H}_{2}$ Inside a Carbon Nanotube. Phys. Rev. B: Condens. Matter Mater. Phys. 2016, 94, 100502.

(32) Bačič, Z.; Light, J. C. Theoretical Methods for Rovibrational States of Floppy Molecules. Annu. Rev. Phys. Chem. 1989, 40, 469.

(33) Echave, J.; Clary, D. C. Potential Optimized Discrete Variable Representation. Chem. Phys. Lett. 1992, 190, 225-230.

(34) Ancilotto, F.; Barranco, M.; Caupin, F.; Mayol, R.; Pi, M. Freezing of ${ }^{4} \mathrm{He}$ and its Liquid-Solid Interface from Density Functional Theory. Phys. Rev. B: Condens. Matter Mater. Phys. 2005, 72, 214522.

(35) Caupin, F.; Ancilotto, F.; Barranco, M.; Mayol, R.; Pi, M. Freezing of Helium-4: Comparison of Different Density Functional Approaches. J. Low Temp. Phys. 2007, 148, 731-736.

(36) Werner, H. J.; Knowles, P. J.; Knizia, G.; Manby, F. R.; Schütz, M.; Celani, P.; Korona, T.; Lindh, R.; Mitrushchenkov, A. O.; Rauhut, G. et al., MOLPRO, version 2012.1, a package of ab initio programs; see http://www.molpro.net.

(37) Heßelmann, A.; Jansen, G.; Schütz, M. Density-Functional Theory-Symmetry-Adapted Intermolecular Perturbation Theory with Density Fitting: A New Efficient Method to Study Intermolecular Interaction Energies. J. Chem. Phys. 2005, 122, 014103.

(38) Perdew, J. P.; Burke, K.; Ernzerhof, M. Generalized Gradient Approximation Made Simple. Phys. Rev. Lett. 1996, 77, 3865-3868.

(39) Woon, D. E.; Dunning, T. H., Jr. Gaussian Basis Sets for Use in Correlated Molecular Calculations. Calculation of Static Electrical Response Properties. J. Chem. Phys. 1994, 100, 2975-2988.

(40) Jenness, G. R.; Jordan, K. D. DF-DFT-SAPT Investigation of the Interaction of a Water Molecule to Coronene and Dodecabenzocoronene: Implications for the Water-Graphite Interaction. J. Phys. Chem. C 2009, 113, 10242-10248.

(41) Weigend, F. A Fully Direct RI-HF Algorithm: Implementation, Optimised Auxiliary Basis Sets, Demonstration of Accuracy and Efficiency. Phys. Chem. Chem. Phys. 2002, 4, 4285-4291.

(42) Weigend, F.; Köhn, A.; Hättig, C. Efficient Use of the Correlation Consistent Basis Sets in Resolution of the Identity MP2 Calculations. J. Chem. Phys. 2002, 116, 3175-3183.

(43) Grüning, M.; Gritsenko, O. V.; van Gisbergen, S. V. A.; Baerends, E. J. Shape Corrections to Exchange-Correlation Potentials by Gradient-Regulated Seamless Connection of Model Potentials for Inner and Outer Region. J. Chem. Phys. 2001, 114, 652-660. 
(44) Lias, S. G. Ionization Energy Evaluation. NIST Chemistry WebBook, NIST Standard Reference Database No. 69; online: http:// webbook.nist.gov/chemistry/.

(45) Adamo, C.; Barone, V. Toward Reliable Density Functional Methods Without Adjustable Parameters: The PBE0Model. J. Chem. Phys. 1999, 110, 6158-6170.

(46) Jeziorski, B.; Moszynski, R.; Ratkiewicz, A.; Rybak, S.; Szalewicz, K.; Williams, H. L. In Mehods and Techniques in Computational Chemistry; METECC94; Clementi, E., Ed.; D. Reidel Publishing Company: STEF, Cagliari, 1993; Vol. B, p 79.

(47) Maroulis, G. Accurate Electric Multiple Moment, Static Polarizability and Hiperpolarizability Derivaties for $\mathrm{N}_{2}$. J. Chem. Phys. 2003, 118, 2673-2687.

(48) Łach, G.; Jeziorski, B.; Szalewicz, K. Radiative Corrections to the Polarizabiliy of Helium. Phys. Rev. Lett. 2004, 92, 233001.

(49) Carlos, W. E.; Cole, M. W. Interaction Between a He Atom and a Graphite Surface. Surf. Sci. 1980, 91, 339.

(50) Brunch, L. W.; Cole, M. C.; Zaremba, E. Physical Adsorption: Forces and Phenomena; Clarendon Press: Oxford, 1997.

(51) Kim, H.-Y.; Cole, M. W.; Mbaye, M.; Gatica, S. Phase Behavior of Ar and Kr Films on Carbon Nanotubes. J. Phys. Chem. A 2011, 115, 7249-7257.

(52) Pernal, K.; Podeszwa, R.; Patkowski, K.; Szalewicz, K. Dispersionless Density Functional Theory. Phys. Rev. Lett. 2009, 103, 263201.

(53) Podeszwa, R.; Szalewicz, K. Density Functional Theory Overcomes the Failure of Predicting Intermolecular Interaction Energies. J. Chem. Phys. 2012, 136, 161102.

(54) Podeszwa, R.; Pernal, K.; Patkowski, K.; Szalewicz, K. Extension of the Hartree-Fock Plus Dispersion Method by First-Order Correlation Effects. J. Phys. Chem. Lett. 2010, 1, 550-555.

(55) Tang, K. T.; Toennies, J. P. An Improved Simple-Model for the van der Waals Potential Based on Universal Damping Functions for the Dispersion Coefficients. J. Chem. Phys. 1984, 80, 3726-3741.

(56) Ruiz, J. C.; Scoles, G.; Jonsson, H. On the Laterally Averaged Interaction Potential Between He Atoms and the (0001) Surface of Graphite. Chem. Phys. Lett. 1986, 129, 139-143.

(57) de Lara-Castells, M. P.; Mitrushchenkov, A. O. Collective Bosonic Excitations in Doped para- $\mathrm{H}_{2}$ Clusters through the FullConfiguration-Interaction Nuclear Orbital Approach. J. Phys. Chem. Lett. 2011, 2, 2145-2151.

(58) de Lara-Castells, M. P.; Delgado-Barrio, G.; Villarreal, P.; Mitrushchenkov, A. O. An Optimized Full-Configuration-Interaction Nuclear Orbital Approach to a Hard-Core Interaction Problem: Application to $\left({ }^{3} \mathrm{He}\right)_{\mathrm{N}}-\mathrm{Cl}_{2}$ (B) Clusters. J. Chem. Phys. 2009, 131, 194101.

(59) de Lara-Castells, M. P.; Delgado-Barrio, G.; Villarreal, P.; Mitrushchenkov, A. O. Microscopic Description of Small Doped ${ }^{3} \mathrm{He}$ Clusters Through the Full-Configuration-Interaction Nuclear Orbital Approach: The $\left({ }^{3} \mathrm{He}\right)_{N^{-}}-\mathrm{Br}_{2}(X)$ Case Revisited. Int. J. Quantum Chem. 2011, 111, 406-415.

(60) Aguirre, N. F.; Villarreal, P.; Delgado-Barrio, G.; Mitrushchenkov, A. O.; de Lara-Castells, M. P. Solvent States and Spectroscopy of Doped Helium Clusters as a Quantum-Chemistry-like Problem. Phys. Chem. Chem. Phys. 2013, 15, 10126-10140.

(61) Svensson, E. C.; Sears, V. F.; Woods, A. D.; Martel, P. Neutrondiffraction Study of the Static Structure Factor and Pair Correlations in Liquid He. Phys. Rev. B: Condens. Matter Mater. Phys. 1980, 21, 36383651. 